\title{
Novelties in Campyloneurum (Polypodiaceae) from Mesoamerica
}

\author{
Alexander Francisco Rojas-Alvarado \\ Universidad Nacional de Costa Rica, Heredia, Costa Rica \\ Email: alfrojasa@yahoo.com
}

How to cite this paper: Rojas-Alvarado, A.F. (2017) Novelties in Campyloneurum (Polypodiaceae) from Mesoamerica. American Journal of Plant Sciences, 8, 921-940. https://doi.org/10.4236/ajps.2017.84062

Received: February 27, 2017

Accepted: March 28, 2017

Published: March 31, 2017

Copyright $\odot 2017$ by author and Scientific Research Publishing Inc. This work is licensed under the Creative Commons Attribution International License (CC BY 4.0).

http://creativecommons.org/licenses/by/4.0/

\begin{abstract}
Three new species are described and another is recognized in Campyloneurum. Two species occur in Mexico to El Salvador and the others two are present in Nicaragua, Costa Rica and Panama. The new species are Campyloneurum acutum A. Rojas, C. leoniae A. Rojas and C. madrense A. Rojas. The first species differs from $C$. xalapense by broader rhizome scales, acute to attenuate blade apex, commonly two series of areoles between parallel veins, commonly one sori per areole and hydathodes absent or few evident. Campyloneurum leoniae differs from $C$. irregulare by more compact and non-pruinose rhizomes, thinner stipes, relatively broader, thinner and more opaque laminae, and distribution at lower elevations. Campyloneurum madrense is different to $C$. angustifolium because it has narrower and darker rhizome scales with denticulate margin, long scales cells and distributed at lower elevations. Finally, Campylonerum pittieri is here recognized as valid species and it differs from the similar $C$. amphostenon by longer rhizomes with appressed scales that have a patent, long acuminate apices, relative broader laminae, veins evident and distribution at higher elevations.
\end{abstract}

\section{Keywords}

Campyloneurum amphostenon Complex, Campyloneurum angustifolium Complex, Campyloneurum xalapense, Lectotype Designed, New Species, Ferns, Species Recognized

\section{Introduction}

Campyloneurum C. Presl is a Neotropical genus with about 50 species [1]. This genus is recognized by having sterile and fertile monomorphic leaves, single, entire and glabrous blades or with few scales on the rachis, sori in 2 - 4 rows between the main lateral veins and they born on the tips of included veinlets [2].

In the Campyloneurum angustifiolium (Sw.) Fée complex, Lellinger described 
C. centrobrasilianum Lellinger, $C$. cooperi Lellinger and $C$. irregulare Lellinger. The first species was compared with $C$. angustipaleatum (Alston) M. Mey. ex Lellinger, the second one with $C$. rigidum J. Sm. and the third with $C$. amphostenon (Kunze ex Klotzsch) Fée. Also he made a new combination from Polypodium angustifolium $\mathrm{f}$. densifolium Hieron. as $C$. densifolium (Hieron.) Lellinger [3]. Of the species previously mentioned, Lellinger registered from Costa Rica to Campyloneurum cooperi and C. irregulare [3]. However, León synonymized this species joining to $C$. angustifolium s. $l$. and mentioned that the group require more studies. Additionally, León registered to $C$. angustipaleatum (Alston) M. Mey. ex Lellinger and C. densifolium from Costa Rica [4].

Lellinger mentioned two distributional ranges from Campyloneurum irregulare in Costa Rica, between 800 - 1500 m and between 2200 - 3300 m [5]. Rojas found that really are two entities with different geographic and altitudinal distribution. Since the plants with the northernmost distribution between Costa Rica and Nicaragua present a non-farinosous rhizome, rhizome scales are with basal cells less markedly irregular and distribute to (700-) 1200 - $1700 \mathrm{~m}$, while the typical form with pruinose rhizome and basal cells of the rhizome scales markedly irregular is located to (1200-) $1700-2500 \mathrm{~m}$. Rojas also mentioned that lowland plants need to merit consideration of variety or species [6].

Mickel \& Smith made difference between $C$. angustifolium and $C$. ensifolium (Willd.) J. Sm., principally referring to rhizome scales, the first with long acuminate apex and with oblong cells, the other with rounded to short acute apex and the cells rounded to squarish [7].

Respect to Campyloneurum xalapense Fée, León mentioned that this taxon has differences in the blade form and variation in rhizome scales between lanceolate to linear, related with populations but not between characters [8]. Mickel \& Smith mentioned different chromosome number between Oaxaca $(=74)$ and Costa Rica (=148) [7], probably due because they are different taxa.

León defined to Campyloneurum amphostenon (Kunze ex Klotzsch) Fée as plants with long-creeping rhizome with dark brown and ovate scales, they are appressed at base, with patent apex [4] [8]. Respect to same species, Rojas found two different entities and separated them in his Table 2, [6], relating entity 1 to the entity proposed by León [4] [8] and entity 2 as a possible hybrid that has short-creeping rhizome with scales 1.5 - 3 times longer than wide and brownyellowish, fronds relatively shorter and narrower, veins few evident and distributed at low elevation.

León [8] mentioned 18 species of Campyloneurum from Mesoamerica, but Rojas with the publication of $C$. gracile and the recognition of $C$. cooperi and $C$. irregulare [6], the inclusion of Hyalotrichopteris anetioides (Christ) W.H. Wagner in Campyloneurum [9] and four species that are included in this work; the current number of species for the region arrives to 26 .

\section{Materials and Methods}

The new species here considered are the result of comparisons with specimens of 
other Mexican and Central American species, and a review of related species and keys from the neotropical ferns by Gómez \& Arbeláez [1], Lellinger [5], Mickel \& Smith [7], Mickel \& Beitel [10], Smith [11] and Stolze [12] and other papers in the Campyloneurum genus [3] [6]. The examined specimens are deposited in the following herbaria CHAPA, CR, MEXU, MO, UC and XAL [13]. To ensure the correct application names, original type material or digital type images were examined as available (Jstor Global Plants (http://plants.jstor.org/)), and the new names were corroborated with International Plant Name Index (http://www.ipni.org/ipni/plantnamesearchpage.do).

\section{Results}

\subsection{New Species}

\subsubsection{Campyloneurum acutum A. Rojas, Sp. Nov. (Figure 1)}

TYPE: MEXICO. Guerrero: Mpio. Atoyac, a $8 \mathrm{~km}$ al SW de Puerto del Gallo, camino a Atoyac, 2140 m, 16 jun 1982, E. Martínez 982 (holotype: MEXU).

Diagnoses: Campyloneurum acutum differs from $C$. xalapense by lanceolate and broader rhizome scales, acute blade apex and parallel veins with commonly two series of areoles.

Description: Epiphytic; rhizome $3-6 \mathrm{~mm}$ in diameter, short-creeping, fronds 2 - $5 \mathrm{~mm}$ distant between them, not pruinose, brown to blackish when dry; rhizome scales 3 - $6 \times 0.5$ - $1.5 \mathrm{~mm}$, lanceolate, brown to yellowish-brown, clathrate or scarcely so with transparent lumina, marginally entire and acute at tip; fronds (20-) 45 - $93 \mathrm{~cm}$ long, moderately decumbent; stipe (3-) 5 - $9 \mathrm{~cm}$ long, 1/12 - 1/9 of the frond length, strawish to pale brown, glabrous, phyllopodia 2 - $3 \mathrm{~mm}$ long; blade (17-) $40-84 \times(2-) 3-4.3(-5.5) \mathrm{cm}$, narrowly elliptic to linear-oblong, gradually reduced at base, long acute to attenuate at apex, chartaceous, lustrous; hydathodes absent or small; rachis strawish to pale brown, glabrous; veins few evident, commonly forming 2 series of areoles between parallel veins, included veinlets one (rarely 2) per areole; sori rounded, in 4 - 6 rows between costa and margin.

Etimology: The name of the new species refers to the acute blade apex.

Distribution: Known from Mexico and El Salvador at $1000-2700 \mathrm{~m}$ elevation.

Additional revised specimens (paratypes): MEXICO. Chiapas: Municipio San Andrés Larrainzar, near the summit of Chuchil Ton, NE of Bochil, $2700 \mathrm{~m}$, 3 Aug 1972, D. Breedlove 26780-A (MEXU); Municipio Motozintla de Mendoza, $45-50 \mathrm{~km}$ NE of Huixtla along road to Motozintla, $1900 \mathrm{~m}, 17$ Nov 1971, D. Breedlove \& A. Smith 22658 (MEXU); SE side of Volcán Tacaná above Talquian, $2200 \mathrm{~m}, 16$ Jan 1973, D. Breedlove \& A. Smith 31631 (MEXU); to $22 \mathrm{~km} \mathrm{E} \mathrm{of}$ Lagunas de Monte Bello, over the trail to Santa Elena, 22 Nov 1982, E. Cabrera \& H. de Cabrera 3687 (MEXU); above El Rosario, $8 \mathrm{mi} \mathrm{S}$ of Motozintla, $1800 \mathrm{~m}, 10$ Jul 1977, T. Croat 40731 (MEXU, MO), ibídem, T. Croat 40732 (MEXU, MO); Municipio Jaltenango, El Triunfo Reserve, trail NNW from El Triunfo camp towards Palo Gordo camp, 1 - $3 \mathrm{~km}$ from El Triunfo camp, $15^{\circ} 39^{\prime} \mathrm{N}, 92^{\circ} 50^{\prime} \mathrm{W}$, 
2000 m, 21 Feb 1990, R. Hampshire et al. 530 (MEXU); Municipio Jaltenango, Reserva El Triunfo, 1 polygon, HQ camp, $15^{\circ} 39^{\prime} \mathrm{N}, 92^{\circ} 48^{\prime} \mathrm{W}, 1850 \mathrm{~m}, 13$ Jun 1990, M. Heath \& A. Long 976 (MEXU); Municipio La Concordia, Cerro Cebú,

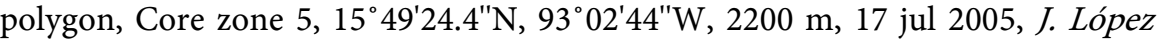
49 (MEXU); Municipio Mapastepec, Triunfo-Tomatal camp, $15^{\circ} 38^{\prime} \mathrm{N}, 92^{\circ} 50^{\prime} \mathrm{W}$, 1728 m, M. López 85 (MEXU); Municipio Unión de Juárez, between Toniná and Talquián, 1700 - 2700 m, 10 May 1987, E. Martínez 20884 (MEXU); Municipio Ziltepec, Pico de Loro range, $8 \mathrm{~km} \mathrm{~S}$ of Nueva Argentina, $15^{\circ} 28^{\prime} 50^{\prime \prime} \mathrm{N}$, 92 36'20"W, 2000 m, 27 Jan 1999, M. Pérez 1739 (MEXU); Municipio Monte Cristo, trail to San Luis, $2 \mathrm{~km}$ from Laguna El Cofre, buffer zone, $15^{\circ} 37^{\prime} 33.8^{\prime \prime} \mathrm{N}$, $92^{\circ} 37^{\prime} 45.1^{\prime \prime W}, 2010$ m, 15 Jun 2005, M. Reynoso 209 (MEXU); Municipio Tapachula, El Dormitorio, hillside W from Volcán Tacaná, 22 May 1984, P. Tenorio et al. 5913 (MO). Guerrero: Municipio Atoyac, 8 km SW from Puerto del Gallo, trail to Atoyac, 2140 m, 16 Jun 1982, E. Martínez 980A (MEXU); Reserva El Triunfo, 1900 m, 1 Jun 1987, E. Martínez et al. 21547 (MEXU); Municipio La Independencia, Colonia Francisco Madero, near the road, 14 Mar 1981, $M$. Sousa et al. 1935 (MEXU); Municipio Yajalón, Surroundings of Ejido Lázaro Cárdenas, $17.21463^{\circ} \mathrm{N}, 92.6059^{\circ} \mathrm{W}, 1152 \mathrm{~m}, 11$ abr 2010, G. Salazar et al. 8904 (MEXU); in Las Golondrinas, $29 \mathrm{~km}$ NE from Paraíso, trail to Puerto del Gallo, 1810 m, 5 Jun 1983, J. Soto et al. 5188 (MEXU); Municipio Zihuatanejo, 86 km NE from Zihuatanejo, by road Zihuatanejo-Ciudad Altamirano, 1530 m, 21 May 1982, P. Tenorio et al. 422 (MEXU). Oaxaca: Municipio Santiago Comaltepec, $2.8 \mathrm{~km}$ in a straight line to $\mathrm{S}\left(225^{\circ}\right)$ from La Esperanza, $17^{\circ} 36^{\prime} 32^{\prime \prime} \mathrm{N}, 96^{\circ} 24^{\prime} 14^{\prime \prime} \mathrm{W}$, 1600 m, 10 Jan 1995, C. Gallardo et al. 1323 (MEXU); Municipio San Felipe Usila, on the periphery of the community of Santa Cruz Tepetotutla, 1000 m, 07 May 1995, M. Romero 2926 (MEXU); Distrito Ixtlán, Municipio Comaltepec, Vista Hermosa, $26.6 \mathrm{~km}$ SW from Valle Nacional, road Tuxtepec-Oaxaca, $17^{\circ} 43^{\prime} \mathrm{N}, 96^{\circ} 20^{\prime} \mathrm{W}, 1460$ m, 26 Jan 1988, R. Torres \& E. Martínez 11417 (MEXU). Queretaro: Municipio Jalpan, 2 - 3 km N from La Parada, 1250 m, 08 Mar 1991, B. Servín 868 (MEXU). Tabasco: Municipio Tacotalpa, Sunú and Patastal, 16 Feb 2000, G. Gaspar \& G. Ocaña 94 (MEXU). Veracruz: Municipio San Andrés Tuxtla, Ejido Barrio Lerdo, slopes of Volcán San Martín Tuxtla, $18^{\circ} 34^{\prime} \mathrm{N}$, 95 10'W, 1000 m, 11 Aug 2005, T. Krömer \& A. Acebey 2474 (MEXU); Municipio San Andrés Tuxtla, Ejido Ruiz Cortines, sidewalk near the town that connects with dirt road, 18³1'59"N, 9508'59"W, $1100 \mathrm{~m}, 25$ May 2001, $A$. Rincón et al. 2427 (MEXU); Municipio Chocaman, Tepejilotla, 1300 m, 29 Jun 1979, F. Ventura 16285 (MEXU).

EL SALVADOR. Santa Ana: Cerro Montecristo, ca. 14 mi NE of Metapán, 1950 - 2000 m, 31 Jul 1977, T. Croat 42399 (MEXU, MO).

Campyloneurum acutum differs from $C$. xalapense by lanceolate (vs. linear or at less with long and narrow apex) and broader apex (0.5 - $1 \mathrm{~mm}$ vs. $0.2-0.5$ $\mathrm{mm}$ ) rhizome scales, acute to attenuate (vs. long acuminate to cuspidate) blade apex, hydathodes absent or small (vs. markerly evident), commonly two (vs. one) series of areoles between parallel veins and commonly one sori per areola 
(vs. 2). Also the new species is related to $C$. tenuipes but differs of it by longer (1/12 - 1/9 of the frond length vs. ca. 1/3) stipe, narrower ((2-) 3 - 4.3 (-5.5) vs. 4 - $9 \mathrm{~cm}$ ) blade, acute to attenuate (vs. long acuminate to cuspidate) blade apex and 4 - 6 (vs. 6 - 10) sori rows between costa and margin (see Figure 1 and Table 1).

\subsubsection{Campyloneurum leoniae A. Rojas, Sp. Nov. (Figure 2)}

TYPE: COSTA RICA. Guanacaste: Cordillera de Tilarán, $1 \mathrm{~km} \mathrm{~N}$ of Las Nubes de Río Chiquito, Zona Monteverde, Atlantic slope, $10^{\circ} 22^{\prime} \mathrm{N}, 84^{\circ} 51^{\prime} \mathrm{W}, 1300 \mathrm{~m}, 4$ Sep 1988, W. Haber \& W. Zuchowski 8691 (holotype: CR!; isotype: MO?).

Diagnoses: Campyloneurum leoniae differs from $C$. irregulare by more compact and non pruinose (vs. pruinose) rhizomes, relatively broader, thinner and more opaque (vs. lustrous) laminae, and distribution at lower elevations.

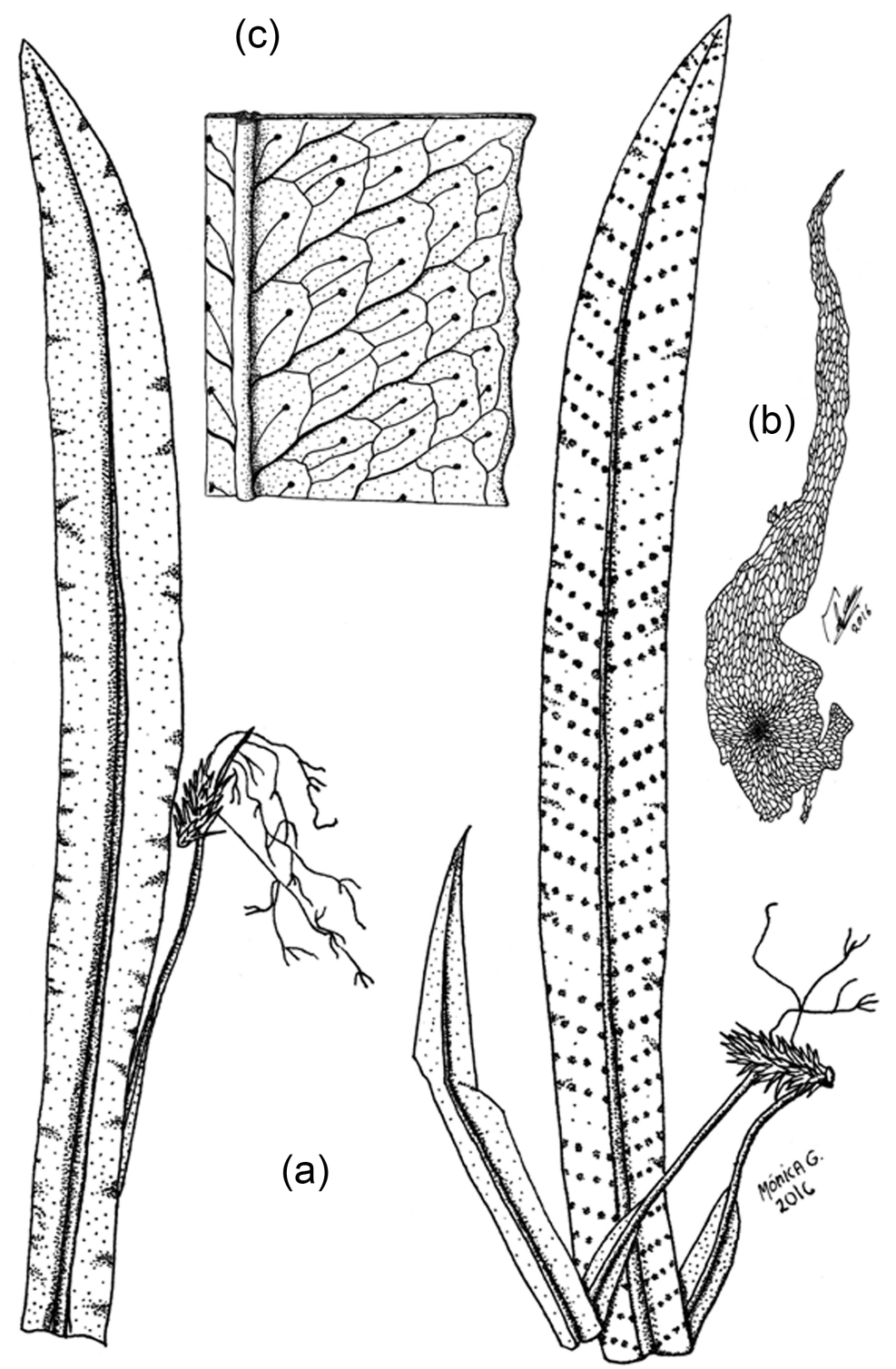

Figure 1. (a)-(b). Type material of Campyloneurum acutum A. Rojas (E. Martínez 982, MEXU). (a) Habit; (b) Rhizome scales; (c) Blade detail. 
Table 1. Comparison of morphological characteristics among three species of Campyloneurum: $C$. acutum, $C$. tenuipes and $C$. xalapense.

\begin{tabular}{|c|c|c|c|}
\hline Character & C. acutum & C. tenuipes & C. xalapense \\
\hline Long of rhizome scales & $3-6 \mathrm{~mm}$ & $5-8 \mathrm{~mm}$ & $3-5 \mathrm{~mm}$ \\
\hline Broad of rhizome scales & $0.5-1.5$ & $1-1.5$ & $0.2-0.5$ \\
\hline Color of rhizome scales & $\begin{array}{c}\text { yellowish-brown to } \\
\text { brown }\end{array}$ & brown & $\begin{array}{l}\text { dark brown to } \\
\text { blackish }\end{array}$ \\
\hline $\begin{array}{l}\text { Lumen color of rhizome } \\
\text { scales }\end{array}$ & translucid & translucid & brown \\
\hline Size of fronds & (20-) $45-93 \mathrm{~cm}$ & $30-80 \mathrm{~cm}$ & (20-) $30-60 \mathrm{~cm}$ \\
\hline Long of stipe & $\begin{array}{c}1 / 12-1 / 9 \text { of the frond } \\
\text { length }\end{array}$ & $\begin{array}{c}1 / 3 \text { of the frond } \\
\text { length }\end{array}$ & $\begin{array}{l}1 / 10 \text { of the frond } \\
\text { length }\end{array}$ \\
\hline Broad of blade & (2-) $3-4.3(-5.5) \mathrm{cm}$ & $4-9 \mathrm{~cm}$ & $3-5.5 \mathrm{~cm}$ \\
\hline Blade apex & acute to attenuate & $\begin{array}{l}\text { acuminate to } \\
\text { cuspidate }\end{array}$ & $\begin{array}{l}\text { acuminate to } \\
\text { cuspidate }\end{array}$ \\
\hline $\begin{array}{l}\text { Rows of areoles between } \\
\text { parallel veins }\end{array}$ & commonly two & two & one \\
\hline $\begin{array}{l}\text { Rows of sori between } \\
\text { midvein and margin }\end{array}$ & $4-6$ & $6-10$ & $4-7$ \\
\hline Altitudinal distribution & $1000-2700 \mathrm{~m}$ & $1000-2300 \mathrm{~m}$ & $\begin{array}{l}500-1700 \\
(-2300) \mathrm{m}\end{array}$ \\
\hline
\end{tabular}

Description: Epiphytic; rhizome 3 - $5 \mathrm{~mm}$ in diameter, short-creeping, fronds approximate to distant to $3 \mathrm{~mm}$, not pruinose, blackish and glabrescent with age; rhizome scales $1.5-3.5(-4) \times 0.8-1.5 \mathrm{~mm}$, ovate-lanceolate to lanceolate, dark brown, clathrate, dense, cordiform at base, cells with irregular base, with the cells at scale apex 1.5 - 3 times longer than wide, lumen pale brown; fronds (8-) $15-35(-45) \mathrm{cm}$ long; stipe $0.5-4(-5) \mathrm{cm}$ long, $1 / 12$ - 1/8 of the frond length, 1 $1.5 \mathrm{~mm}$ wide (to $2 \mathrm{~mm}$ with wing), slight to strongly flattened, strawish to pale brown, winged throughout, glabrous or sparsely scaly, the scales $0.5-1.5 \mathrm{x}$ ca. $0.5 \mathrm{~mm}$, ovate, brown; phyllopodia 0.5 - 1 (-2) mm long; blade (7-) 13 - 30 (-40) $\times(0.5-) 1-2(-2.3) \mathrm{cm}$, linear-elliptic, attenuate on both sides, coriaceous, opaque, with commonly revolute margin; rachis strawish to brown, glabrous or sparsely scaly at base; lateral veins few evident; sori rounded, in 1 - $3(-4)$ irregular rows between costa and margin.

Etimology: This new species is dedicated to Blanca León, Peruvian pteridologist, who has made several contributions to Campyloneurum studies.

Distribution: Known from Nicaragua and Costa Rica at $700-1800(-2000) \mathrm{m}$ elevation.

Additional revised specimens (paratypes): NICARAGUA. Estelí: Cerro

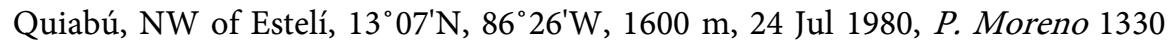
(CR, HNMN); foothill of Quiabú, side N, $13^{\circ} 06^{\prime} \mathrm{N}, 86^{\circ} 25^{\prime} \mathrm{W}, 1460-1480 \mathrm{~m}, 2 \mathrm{Jul}$ 1982, P. Moreno 16775 (CR, HNMN). Granada: Volcán Mombacho, 1345 m, 31 Jan 1980, M. Araquistain \& P. Moreno 1206 (CR, HNMN). Jinotega: Santa Gertrudis, $30 \mathrm{~km}$ E of Jinotega, $1000 \mathrm{~m}, 20$ Jan 1980, M. Araquistain \& $P$. Moreno 867 (CR, HNMN); ca. $23.5 \mathrm{~km}$ (by road) NE of hwy. 1 at Estelí and ca. $2.8 \mathrm{~km}$ SE of Laguna Miraflores, ca. $13^{\circ} 14^{\prime} \mathrm{N}, 86^{\circ} 14^{\prime} \mathrm{W}, 1400 \mathrm{~m}, 11$ Jun 1981, J. Henrich \& W. Stevens 408 (CR, MO); ibídem, J. Henrich \& W. Stevens 410 (CR, 
$\mathrm{MO})$; along hwy 3, ca. $1.9 \mathrm{~km} \mathrm{NW}$ of Aranjuez, road to entrance, ca. $13^{\circ} 02^{\prime} \mathrm{N}$, 85 56'W, 1460 - 1480 m, 24 Dec 1977, W. Stevens \& B. Krukoff 5537 (CR, MO); ca. $0,6 \mathrm{~km}$ from hwy 3 on road to Aranjuez, ca. $13^{\circ} 01^{\prime} \mathrm{N}, 85^{\circ} 55^{\prime} \mathrm{W}$, ca. $1430 \mathrm{~m}, 9$ Apr 1978, W. Stevens \& B. Krukoff 7543 (CR, MO); along hwy 3, ca $1 \mathrm{~km} \mathrm{NW}$ of La Fundadora entrance, unnamed peak ca. $500 \mathrm{~m} \mathrm{~W}$ of hwy, ca. $13^{\circ} 01^{\prime} \mathrm{N}$, 85 56'W, 1450 - 1520 m, 24 May 1981, W. Stevens et al. 20391 (CR); Miraflores, Aug 1975, J. Villa s.n. (CR). Madriz: Cerro Pataste, ca. 20 km SW of Somoto city, ca. 1700 m, 28 Aug 1982, A. Grijalva 897 (CR, HNMN). Matagalpa: La Carlota, road Matagalpa-Tuma, 1400 - 1500 m, Oct 1975, L. Gómez et al. 6298 (CR); Fuente Pura, Santa María de Ostuma, between Matagalpa and Jinotega, $1400 \mathrm{~m}$, 26 Aug 1982, E. Martínez et al. 1731 (MEXU); trail to Cerro La Carlota, $2 \mathrm{~km}$ of the trail to Tuma, $12^{\circ} 58^{\prime} \mathrm{N}, 85^{\circ} 52^{\prime} \mathrm{W}, 1040-1100 \mathrm{~m}, 5$ Mar 1982, P. Moreno 15618 (CR, HNMN); Santa María de Ostuma estate, 10 km N of Matagalpa, 1300 m, 17 Jul 1978, P. Vincelli 755 (CR).

COSTA RICA. Alajuela: Piedades Norte (Los Ángeles) of San Ramón, 6 Mar 1929, A. Brenes 6724 (CR); La Palma of San Ramón, 2 Nov 1929, A. Brenes 7396 (145) (CR); San Pedro of San Ramón, 31 Jan 1936, A. Brenes 21558 (8) (CR); San Carlos, 14 km NE of Ciudad Quesada, 1250 m, 22 Jul 1963, A. Jiménez 950 (CR); above Angel Falls, Río La Paz Grande, on road to Sarapiquí, 1460 m, 23 Mar 1969, R. Lent 1501 (CR); above Río Toro, 10²1'N, 84¹9'W, 1550 m, 3 Sep 1972, R. Lent 2812 (CR); La Palma of San Ramón, 3 - 10 Aug 1935, M. Quirós 259 (CR); Carrillos de Poás, 23 Aug 1936, M. Quirós 569 (CR); San Ramón, Reserva Forestal San Ramón, Cordillera de Tilarán, Los Ángeles, banks of the road between Los Ángeles and Río Cataratas, 10¹2'15"N, 84³2'15"W, 900 - 1250 m, 2 May 1996, A. Rojas \& J. Morales 2589 (CR). Cartago: Pacayas, near of bridge over the Río Turrialba, trail to Turrialba (between Santa Teresa and Santa Cruz), $9^{\circ} 57^{\prime} 20^{\prime \prime N}, 83^{\circ} 46^{\prime} 20^{\prime \prime} \mathrm{W}, 1670 \mathrm{~m}, 27$ Oct 1995, A. Cascante et al. 847 (CR); $10 \mathrm{~km}$ $\mathrm{S}$ of Cartago by air, along confluence of río Empalme and Río Estrella, $1 \mathrm{~km} \mathrm{~S}$ of Palo Verde by road, Palo Verde is $1 \mathrm{~km} \mathrm{~S}$ of Panamerican hwy on road to La Estrella, 946'N, 835' W, 1450 m, 21 Apr 1983, R. Liesner \& E. Judziewicz 14587 (MO); Paraíso, Cordillera de Talamanca, Estación de Biología Tropical Río Macho and surroundings, Orosi, 09 $46^{\prime} \mathrm{N}, 83^{\circ} 52^{\prime} \mathrm{W}, 1550-1580 \mathrm{~m}, 30$ Sep 1993, $A$. Rojas 480 (CR, INB, MO); Guadalupe (Arenilla), Zona Protectora La Carpintera, slope of hill of La Carpintera, with view to Coris, 9 $52^{\prime} 39.2^{\prime \prime} \mathrm{N}, 83^{\circ} 58^{\prime} 17.6^{\prime \prime} \mathrm{W}$, 1710 m, 19 Mar 2007, A. Ruiz et al. 1179 (CR); $0.8 \mathrm{~km}$ down (W) road to San Cristobal Norte, 1850 m, 21 Set 1979, K. Walter 79498 (CR). Guanacaste: La Cruz, Parque Nacional Guanacaste, $9 \mathrm{~km} \mathrm{~S}$ of Santa Cecilia, Estación Pitilla, $10^{\circ} 59^{\prime} 26^{\prime \prime} \mathrm{N}, 85^{\circ} 25^{\prime} 40^{\prime \prime} \mathrm{W}, 700$ m, 28 Sep 1990, C. Chávez et al. 188 (CR, MO); Monteverde, $3.4 \mathrm{~km} \mathrm{~N}$ of Santa Elena on road to Las Nubes, $10^{\circ} 22^{\prime} \mathrm{N}, 84^{\circ} 49^{\prime} \mathrm{W}$, 1500 m, 14 Aug 1988, W. Haber \& W. Zuchowski 8547 (CR, MO); Monteverde, $3.5 \mathrm{~km}$ N of Santa Elena on road to San Gerardo, $0.5 \mathrm{~km}$ N of junction road and Río Negro, $10^{\circ} 21^{\prime} \mathrm{N}, 84^{\circ} 48^{\prime} \mathrm{W}, 1540$ m, 20 Aug 1988, W. Haber \& W. Zuchowski 8579 (CR); Cordillera de Tilarán, $1 \mathrm{~km}$ N of Las Nubes de Río Chiquito, Monteverde zone, Atlantic slope, $10^{\circ} 22^{\prime} \mathrm{N}, 84^{\circ} 51^{\prime} \mathrm{W}, 1300 \mathrm{~m}, 4$ Sep 1988, W. Haber \& W. Zuchowski 8689 (CR, MO); Tilarán, Cordillera de Tilarán, Río San Lucas, 
$10^{\circ} 22^{\prime} 50^{\prime \prime N}, 84^{\circ} 54^{\prime} 20^{\prime \prime W}, 800$ - 900 m, 27 Jun 1995, G. Rodríguez \& A. Rojas 357 (CR); La Unión, San Rafael, Zona Protectora Cerros La Carpintera, Iztarú camp, between the road and the top of the mountain, $9^{\circ} 53^{\prime} 11^{\prime \prime} \mathrm{N}, 83^{\circ} 58^{\prime} 16^{\prime \prime} \mathrm{W}, 1750$ 1850 m, 17 Jul 2007, A. Rojas et al. 7599 (CR). Heredia: Vara Blanca, 1700 - 1900 m, 27 Oct 1979, C. Todzia 929 (CR). Limón: Talamanca, trail ca. $5 \mathrm{~km}$ up Alto Lari, 9 $24^{\prime} 15^{\prime \prime} \mathrm{N}, 83^{\circ} 05^{\prime} 05^{\prime \prime} \mathrm{W}, 1300$ m, 27 Feb 1992, J. Bittner 1338 (CR); Talamanca, Bratsi, Amubri, Alto Lari, Kivut, 9²4'15"N, 8305'15"W, 1300 m, 6 Mar 1992, G. Herrera 5174 (CR). Puntarenas: Coto Brus, Parque Internacional La Amistad, Cordillera de Talamanca, Estación Pittier, Río Gemelo trail, 9 $01^{\prime} 30^{\prime \prime} \mathrm{N}$, $82^{\circ} 57^{\prime} 40^{\prime \prime W}, 1680$ m, 29 Jan 1995, F. Alvarado et al. 12 (INB, CR); upper río Burú, 2010 m, 19 Aug 1983, L. Gómez et al. 21431 (CR); Ojo de Agua, Monteverde, Méndez estate, Río Aranjuez, $10^{\circ} 17^{\prime} \mathrm{N}, 84^{\circ} 46^{\prime} \mathrm{W}, 1550 \mathrm{~m}, 14$ Nov 1987, W. Haber \& E. Bello 7731a (CR); La Pitahaya, Arancibia, Rincón, headers of Río Aranjuez, $10^{\circ} 15^{\prime} 10^{\prime \prime} \mathrm{N}, 84^{\circ} 41^{\prime} 20^{\prime \prime} \mathrm{W}, 1200$ m, 27 May 1996, G. Herrera 9024 (CR); Monteverde Reserve, $1 \mathrm{~km} \mathrm{SW}$ station, $10^{\circ} 18^{\prime} \mathrm{N}, 84^{\circ} 48^{\prime} \mathrm{W}, 1500$ 1550 m, 20 Feb 1992, S. Ingram \& K. Ingram 1361 (CR); Monteverde, 1520 m, 13 Nov 1979, S. Koptur 223 (CR); Monteverde, 1 Dec 1979, S. Koptur 246 (CR); Monteverde, Cordillera de Tilarán, 1350 m, 30 Jul 1981, D. Neill 5067 (CR); Monteverde, Cordillera de Tilarán, $4500 \mathrm{ft}$ [=1372 m], Mar 1959, C. Palmer 110 (CR); Coto Brus, Zona Protectora Tablas, Cordillera de Talamanca, Sabalito, Las Alturas de Cotón, Estación Biológica Las Alturas, surroundings of the station, $8^{\circ} 57^{\prime} 15^{\prime \prime} \mathrm{N}, 82^{\circ} 50^{\prime} 10^{\prime \prime} \mathrm{W}, 1580 \mathrm{~m}, 23$ Dec 1993, A. Rojas 765 (INB, MO); Monteverde Cloud Forest Reserve, road to station from comunity, $10^{\circ} 06^{\prime} \mathrm{N}$, 83²6'W, 1500 m, 23 Aug 1987, J. Smith 563 (CR). San José: Pérez Zeledón, Cordillera de Talamanca, Las Nubes de Santa Elena, 9²3'30"N, 83³5'50"W, 1210 m, 8 sep 1995, E. Alfaro 395 (CR, INB); Desamparados, Patarrá, Cerro El Espino (Mata de Caña-Azahar), 1600 - 1800 m, 13 Nov 1983, I. Chacón \& G. Herrera 1564 (CR); ibídem, I. Chacón \& G. Herrera 1569 (CR); Coronado, Las Nubes, 29 Dec 1956, F. Chisaki 1004 (CR); surroudings S of San José, trail more than $1 \mathrm{~km}$ NE of Tarbaca to Joya, which deviates from the road no. 4 Aserrí-Tarbaca,

1750 m, 29 Apr 1990, P. Döbbeler 112 (MEXU); Tarrazú, San Carlos, trail to San Josecito, 9 $37^{\prime} 20^{\prime \prime} \mathrm{N}, 84^{\circ} 07^{\prime} 30^{\prime \prime W}, 1400$ m, 23 set 1997, A. Estrada 1177 (CR); Escazú, cerros de Escazú, to Alto Tapezco by Bebedero, 9 ${ }^{\circ} 55^{\prime} 00^{\prime \prime} \mathrm{N}, 84^{\circ} 10^{\prime} 00^{\prime \prime} \mathrm{W}$, 1100 - 1700 m, 3 Oct 1993, B. Hammel et al. 19070 (CR); Dota, Peor es Nada, $\mathrm{NW}$ from the foothills of Cerro Lira, 9 $31^{\prime} 30^{\prime \prime} \mathrm{N}, 83^{\circ} 51^{\prime} 40^{\prime \prime} \mathrm{W}, 1800 \mathrm{~m}, 8 \mathrm{Dec}$ 1994, G. Herrera \& S. Martén 7410 (CR); Dota, Copey, Providencia, Zapotal, path on the right after the river, $9^{\circ} 31^{\prime} 50^{\prime \prime} \mathrm{N}, 83^{\circ} 49^{\prime} 49^{\prime \prime} \mathrm{W}, 1800 \mathrm{~m}, 20$ Aug 2003, $S$. Lobo \& A. Ruiz 507 (CR); Aserrí, Cerros de Escazú, Tarbaca, 949'10"N, $84^{\circ} 07^{\prime} 30^{\prime \prime} \mathrm{W}, 1850$ m, 12 Oct 1993, J. Morales 1871 (CR); Parque Nacional Braulio Carrillo, ca. $1 \mathrm{~km}$ along road from entrance, $1500 \mathrm{~m}$, 19 Jul 1983, R. Moran 3294 (CR, F); Dota, Copey, Zapotal de Providencia, of the Montaña Fría farm 0 - $1 \mathrm{~km}$ to Providencia, 9 $32^{\prime} 05^{\prime \prime} \mathrm{N}, 83^{\circ} 50^{\prime} 19^{\prime \prime} \mathrm{W}, 1720 \mathrm{~m}, 26$ Aug 2004, A. Rojas \& H. Gómez 5975 (CR, MO); Tarrazú, San Carlos, Cerro Cura, trail to San Marcos, $9^{\circ} 36^{\prime} 17^{\prime \prime} \mathrm{N}, 84^{\circ} 06^{\prime} 39^{\prime \prime W}, 1730$ m, 21 Sep 2004, A. Rojas \& S. Lobo 6096 (CR); 
Aserrí, Cerros de Escazú-La Carpintera, Tarbaca, El Cedral, trail to Pico Alto, $9^{\circ} 49^{\prime} 35^{\prime \prime N}, 84^{\circ} 06^{\prime} 50^{\prime \prime W}, 1770$ - 2000 m, 16 Jun 1996, A. Rojas \& M. Obando 2628 (CR); Acosta, Palmichal, Zona Protectora Cerros de Escazú, side of Río Tabarcia, $9^{\circ} 50^{\prime} 32^{\prime \prime} \mathrm{N}, 84^{\circ} 10^{\prime} 21^{\prime \prime} \mathrm{W}, 1450 \mathrm{~m}, 23$ Jun 2004, A. Rojas \& A. Quesada 5789 (CR); along Río Zurquí, (downstream from Guápiles hwy), Cordillera Central, $10^{\circ} 03^{\prime} \mathrm{N}, 84^{\circ} 0^{\prime} \mathrm{W}$, ca. 1400 - $1500 \mathrm{~m}, 18$ Jan 1986, A. Smith et al. 1731 (CR, UC); Escazú, Piedra Blanca, 1900 m, 20 Jan 1935, F. Solís 149 (CR); San Vicente, La Justa, 1170 m, Sep 1925, M. Valerio 124 (CR).

Campyloneurum leoniae separates from $C$. irregulare because has not pruinose rhizome (vs. pruinose); fronds approximate to $3(-5) \mathrm{mm}$ distant (vs. (approximate-) 2 - $5 \mathrm{~mm}$ distant); rhizome scales with the apical cells 2 - 5 timers longer tan wide (vs. 1.5 - 3.5 times); phyllopodia relatively shorter $(0.5-1(-2)$ $\mathrm{mm}$ vs. 1 - $3 \mathrm{~mm}$ ); adaxial blade surface opaque (vs. lustrous (rarely opaque)) and distributed to lower elevation ((700-) $1000-1850$ (-2000) m vs. (1700-) 190 - $2500 \mathrm{~m}$ ) (See Figure 2 and Table 2).

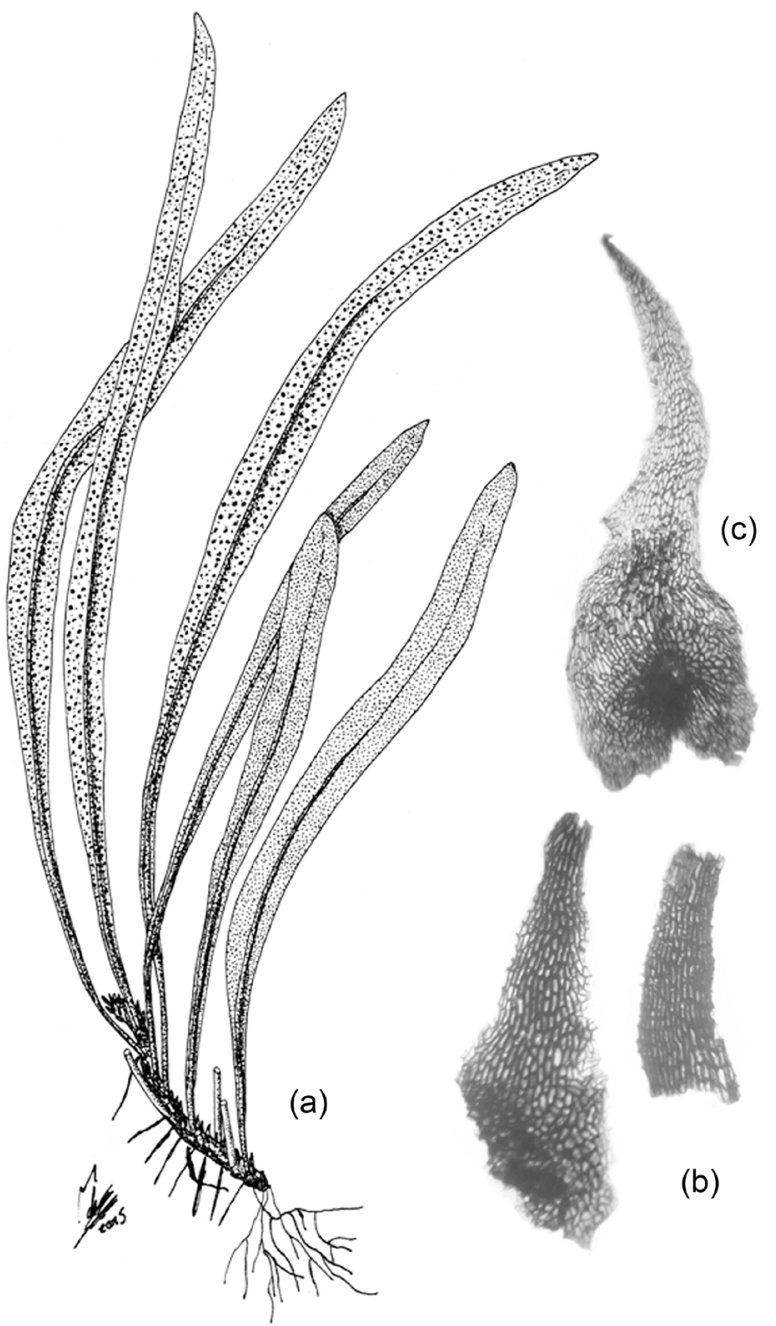

Figure 2. (a) (b). Type material of Campyloneurum leoniae A. Rojas (W. Haber \& W. Zuchowski 8691, CR). (a) Habit; (b) Rhizome scales; (c) Rhizome scales of Campyloneurum irregulare Lellinger (A. Rojas et al. 6229, CR). 
Table 2. Comparison of morphological characteristics among four species of Campyloneurum: C. amphostenon, C. irregulare, C. leoniae and C. pittieri.

\begin{tabular}{|c|c|c|c|c|}
\hline Character & C. amphostenon & C. irregulare & C. leoniae & C. pittieri \\
\hline $\begin{array}{l}\text { Rhizome } \\
\text { glaucous }\end{array}$ & yes & yes & no & No or scarcely \\
\hline $\begin{array}{l}\text { Distance } \\
\text { between } \\
\text { fronds }\end{array}$ & $2-15 \mathrm{~mm}$ & $\begin{array}{l}\text { (approximate-) } 2 \\
-5 \mathrm{~mm} \text { distant }\end{array}$ & $\begin{array}{c}\text { Approximate } \\
\text { to } 3(-5) \mathrm{mm} \\
\text { distant }\end{array}$ & $5-30(-50) \mathrm{mm}$ \\
\hline $\begin{array}{c}\text { Long of } \\
\text { rhizome scales }\end{array}$ & $4-8 \mathrm{~mm}$ & $3-5 \mathrm{~mm}$ & $1.5-4.0 \mathrm{~mm}$ & $2-5 \mathrm{~mm}$ \\
\hline $\begin{array}{c}\text { Color of } \\
\text { rhizome scales }\end{array}$ & $\begin{array}{l}\text { yellowish-brown } \\
\text { to grayish-brown }\end{array}$ & $\begin{array}{c}\text { brown to dark } \\
\text { brown }\end{array}$ & $\begin{array}{c}\text { brown to dark } \\
\text { brown }\end{array}$ & $\begin{array}{l}\text { brown to dark } \\
\text { brown }\end{array}$ \\
\hline $\begin{array}{c}\text { Form of basal } \\
\text { cell of } \\
\text { rhizome scales }\end{array}$ & $\begin{array}{l}\text { triangular to } \\
\text { hexagonal, } \\
\text { isodiametric }\end{array}$ & $\begin{array}{l}\text { poligonal, } \\
\text { irregular }\end{array}$ & $\begin{array}{l}\text { poligonal, } \\
\text { irregular }\end{array}$ & $\begin{array}{l}\text { Rounded to } \\
\text { poligonal, } \\
\text { isodiametric }\end{array}$ \\
\hline $\begin{array}{l}\text { Long of apical } \\
\text { cells of } \\
\text { rhizome scales }\end{array}$ & $\begin{array}{l}1.5 \text { - } 3.0 \text { times } \\
\text { longer than wide }\end{array}$ & $\begin{array}{l}1.5 \text { - } 3.5 \text { times } \\
\text { longer than wide }\end{array}$ & $\begin{array}{c}2 \text { - } 5 \text { times } \\
\text { longer than } \\
\text { wide }\end{array}$ & $\begin{array}{c}1 \text { - } 2 \text { times } \\
\text { longer than wide }\end{array}$ \\
\hline $\begin{array}{c}\text { Lumen cells } \\
\text { color of } \\
\text { rhizome scales }\end{array}$ & translucid & brown & brown & $\begin{array}{l}\text { translucid to } \\
\text { grayish-brown }\end{array}$ \\
\hline Fronds size & $30-55(-65) \mathrm{cm}$ & $20-45 \mathrm{~cm}$ & $\begin{array}{l}(10-) 20-35 \\
(-50) \mathrm{cm}\end{array}$ & $\begin{array}{l}(35-) 45-60 \\
(-80) \mathrm{cm}\end{array}$ \\
\hline $\begin{array}{c}\text { Long of } \\
\text { phyllopodia }\end{array}$ & $1.5-3 \mathrm{~mm}$ & $1-3 \mathrm{~mm}$ & $0.5-1(-2) \mathrm{mm}$ & $2-4 \mathrm{~mm}$ \\
\hline Long of stipe & $4-10(-20) \mathrm{cm}$ & $\begin{array}{c}0.5-3.0(-5.0) \\
\mathrm{cm}\end{array}$ & $\begin{array}{c}(0.5-) 1-5.0 \\
\mathrm{~cm}\end{array}$ & $8-25 \mathrm{~cm}$ \\
\hline $\begin{array}{l}\text { Diameter of } \\
\text { stipe }\end{array}$ & $2-3 \mathrm{~mm}$ & (1-) $1.5-2 \mathrm{~mm}$ & $1-1.5 \mathrm{~mm}$ & $2-3 \mathrm{~mm}$ \\
\hline Broad of blade & $1.0-3.5 \mathrm{~cm}$ & $\begin{array}{c}(0.4-) 0.6-1.2 \\
\mathrm{~cm}\end{array}$ & $\begin{array}{c}(0.5-) 1-2(-.3) \\
\mathrm{cm}\end{array}$ & $\begin{array}{c}(1.3-) 2.0-5.0 \\
\mathrm{~cm}\end{array}$ \\
\hline $\begin{array}{l}\text { Brightness of } \\
\text { abaxial blade } \\
\text { surface }\end{array}$ & $\begin{array}{l}\text { middle to markerly } \\
\text { lustrous }\end{array}$ & $\begin{array}{c}\text { lustrous (rarely } \\
\text { opaque) }\end{array}$ & opaque & middle lustrous \\
\hline $\begin{array}{l}\text { Highlighted } \\
\text { veins }\end{array}$ & few or not & not & not & yes \\
\hline $\begin{array}{l}\text { Altitudinal } \\
\text { distribution }\end{array}$ & $\begin{array}{l}(1800-) 2100- \\
2900(-3200) \mathrm{m}\end{array}$ & $\begin{array}{c}(1700-) 1900- \\
2500 \mathrm{~m}\end{array}$ & $\begin{array}{c}(700-) 1000- \\
1850(-2000) \mathrm{m}\end{array}$ & $\begin{array}{c}(1900-) 2700- \\
3400(-3550) \mathrm{m}\end{array}$ \\
\hline
\end{tabular}

\subsubsection{Campyloneurum madrense A. Rojas, Sp. Nov. (Figure 3).}

TYPE: MEXICO. Chiapas: Mpio. Ocosingo, $6.35 \mathrm{~km} \mathrm{NW}$ of San Javier cruise, $16^{\circ} 50^{\prime} 34^{\prime \prime} \mathrm{N}, 91^{\circ} 23^{\prime} \mathrm{W}, 391 \mathrm{~m}, 15 \mathrm{Nov} 2003$, D. Alvarez et al. 6960 (holotype: MEXU).

Diagnoses: Campyloneurum madrense differs from C. angustifolium by not pruinose rhizome or scarcely so, linear rhizome scales with sparsely denticulate margin, they dark gray to blackish, inside with narrow oblong cells and distributed at lower altitudinal distribution.

Description: Epiphytic; rhizome $2-5 \mathrm{~mm}$ in diameter, compact to short-creeping, fronds approximate to distant to $2 \mathrm{~mm}$, not pruinose, brown to blackish when dry; rhizome scales $3-6 \times 0.4-0.8 \mathrm{~mm}$, linear to linear-lanceolate, dark gray to blackish, clathrate, dense, apically long acute to attenuate, marginally sparsely denticulate, cells oblong to narrowly oblong, 2 - 5 times longer than wide, lumen gray; fronds (18-) 30 - $65 \mathrm{~cm}$ long; stipe 2 - 5 (-8) 
cm long, 1/12 - 1/8 of the frond length, $1-1.5 \mathrm{~mm}$ wide (to $2 \mathrm{~mm}$ with wing), slight to strongly flattened, strawish to pale brown, winged throughout, glabrous or sparsely scaly, the scales $0.5-1.5 \mathrm{x}$ ca. $0.5 \mathrm{~mm}$, ovate, brown; phyllopodia 0.5 1 (-2) mm long; blade (16-) $27-48 \times(0.5-) 0.8-1.5 \mathrm{~cm}$, linear, attenuate on both sides, chartaceous to subcoriaceous, opaque to lustrous, with commonly revolute margin; rachis strawish to brown, glabrous or sparsely scaly at base; lateral veins few evident; sori rounded, in 1 - 2 (-3) irregular rows between costa and margin.

Etimology: The name of it species make reference to geographical distribution in Sierra Madre Oriental and Sierra Madre del Sur.

Distribution: Actually known only from Mexico at 25 - 940 m elevation.

Additional revised specimens (paratypes): MEXICO. Chiapas: Municipio Ocosingo, $2 \mathrm{~km} \mathrm{~S}$ of Nuevo Guerrero, $16^{\circ} 59^{\prime} 02^{\prime \prime} \mathrm{N}, 91^{\circ} 17^{\prime} 07^{\prime \prime} \mathrm{W}, 248 \mathrm{~m}$, 31 Jan 2003, G. Aguilar 5371 (MEXU); Municipio Ocosingo, $0.83 \mathrm{~km}$ SE of Nuevo Guerrero village, $16^{\circ} 58^{\prime} 51^{\prime \prime N}, 91^{\circ} 16^{\prime} 44^{\prime \prime} \mathrm{W}, 222$ m, 4 Nov 2003, G. Aguilar 8347 (MEXU); Municipio Ocosingo, $4.2 \mathrm{~km}$ E of Nuevo Francisco León, 16 $16^{\circ} 43^{\prime \prime} \mathrm{N}$, $91^{\circ} 16^{\prime} 57^{\prime \prime} \mathrm{W}, 180$ m, 13 Oct 2002, G. Aguilar \& D. Alvarez 3443 (MEXU); Municipio Ocosingo, $6.37 \mathrm{~km} \mathrm{SE}$ of Nuevo Guerrero, trail of Lancanjá-Tzeltal, $16^{\circ} 55^{\prime} 57^{\prime \prime} \mathrm{N}, 91^{\circ} 15^{\prime} 43^{\prime \prime} \mathrm{W}, 456 \mathrm{~m}, 15$ Oct 2002, G. Aguilar \& D. Álvarez 3575 (MEXU) Municipio Ocosingo, $1.3 \mathrm{~km} \mathrm{~S}$ of El Paraíso, $16^{\circ} 57^{\prime} 05^{\prime \prime} \mathrm{N}, 91^{\circ} 15^{\prime} 43^{\prime \prime} \mathrm{W}$, 483 m, 16 Oct 2002, G. Aguilar \& D. Álvarez 3642 (MEXU); Municipio Ocosingo, $0.09 \mathrm{~km}$ SO of Lacanjá Chansayab community, $16^{\circ} 45^{\prime} 39^{\prime \prime} \mathrm{N}, 91^{\circ} 07^{\prime} 47^{\prime \prime} \mathrm{W}, 325$ m, 15 Nov 2003, G. Aguilar \& C. Chancayun 8425 (MEXU); Municipio Ocosingo, waterhole of San Javier, $16^{\circ} 50^{\prime} 25^{\prime \prime} \mathrm{N}, 91^{\circ} 08^{\prime} 32^{\prime \prime} \mathrm{W}, 390 \mathrm{~m}, 2$ Dec 2002, G. Aguilar \& A. Cortés 4504 (MEXU); Municipio Ocosingo, Chansayab community, $16^{\circ} 45^{\prime} 16^{\prime \prime N}, 91^{\circ} 07^{\prime} 55^{\prime \prime W}, 362 \mathrm{~m}, 19$ Oct 2002, G. Aguilar et al. 3830 (MEXU), ibídem, G. Aguilar et al. 3832 (MEXU); Municipio Ocosingo, $4.8 \mathrm{~km} \mathrm{SE}$ of Nuevo Guerrero, $16^{\circ} 57^{\prime} 26^{\prime \prime N}, 81^{\circ} 15^{\prime} 02^{\prime \prime} \mathrm{W}, 338 \mathrm{~m}, 23$ May 2002, D. Álvarez \& G. Aguilar 1420 (MEXU); Municipio Ocosingo, $4.5 \mathrm{~km}$ SE of Bonampak archaeological zone, $16^{\circ} 40^{\prime} 33^{\prime \prime} \mathrm{N}, 91^{\circ} 02^{\prime} 05^{\prime \prime} \mathrm{W}, 256 \mathrm{~m}, 10 \mathrm{Feb} 2003, D$. Álvarez \& A. Chambor 3720 (MEXU); Municipio Ocosingo, $7.5 \mathrm{~km} \mathrm{SW}$ of Bonampak, $16^{\circ} 39^{\prime} 51^{\prime \prime N}, 91^{\circ} 00^{\prime} 59^{\prime \prime} \mathrm{W}, 25 \mathrm{~m}, 28$ Nov 2002, D. Alvarez et al. 2743 (MEXU) Municipio Ocosingo, 6,35 km NW of the cruise of San Javier, $16^{\circ} 34^{\prime} \mathrm{N}$, $91^{\circ} 08^{\prime} 43^{\prime \prime} \mathrm{W}, 391 \mathrm{~m}, 15 \mathrm{Nov}$ 2003, D. Álvarez et al. 6960 (MEXU); Municipio Ocozocoautla de Espinoza, 26 - $28 \mathrm{~km} \mathrm{~N}$ of Ocozocoautla, along road to Mal Paso, 700 m, 15 Nov 1971, D. Breedlove \& A. Smith 22445 (MEXU); $13 \mathrm{mi} \mathrm{N}$ of Ocozocoautla, on gravel road to Apitpac, near km 21 marker, 900 m, 9 Jul 1977, T. Croat 40605 (MEXU, MO); along dirt road between San Fernando and Moravillas (near Lago Malpaso), 4 - $66 \mathrm{mi} \mathrm{NW}$ of San Fernando, $16^{\circ} 53^{\prime} \mathrm{N}, 93^{\circ} 16^{\prime} \mathrm{W}$, 840 - 940 m, 15 Feb 1987, T. Croat \& D. Hannon 65024 (MEXU, MO); along hwy 197, between Chiapa de Corzo and Pichucalco, $0.6 \mathrm{mi} \mathrm{N}$ of Tapulula, 35.6 mi SSE of Pichucalco, vicinity of km 124 marker, $17^{\circ} 16^{\prime} \mathrm{N}, 93^{\circ} 01^{\prime} \mathrm{W}, 750 \mathrm{~m}, 18$ Feb 1987, T. Croat \& D. Hannon 65272 (MEXU, MO); Municipio Ocosingo, 4 $\mathrm{km}$ SE of Nuevo Guerrero, trail to Boca Lacantum, 560 m, 24 Jan 1986, E. 
Martínez 16764 (MEXU); Municipio Ocozocoautla, Reserva Ecológica "El Ocote", 750 m, 17 Feb 1986, M. Palacios 2884 (MEXU, UC, XAL); Municipio Ocosingo, Río Cedro, W of Lacanja Chansayab, 300 m, 15 Jan 1984, F. Vázquez \& S. Avendaño 1514 (MEXU, XAL). Oaxaca: Municipio San Pedro Ixcatlan, In the village of Cerro Quemado, road entrance Ojitlán-Xalapa de Diaz, $230 \mathrm{~m}, 8$ Feb 1984, J. Calzada 10263 (UC, XAL); Municipio Soyaltepec, Distrito Tuxtepec, spillway of the Temascal dam, $18^{\circ} 12^{\prime} \mathrm{N}, 96^{\circ} 20^{\prime} \mathrm{W}, 70 \mathrm{~m}, 21$ oct 1987, L. Cortes \& $R$. Torres 1092 (MEXU); along road from Valle Nacional to Arriba Tortuga and Armadillo, via Rancho Grande and Loma San Rafael, 5 - 6 km NW of Valle

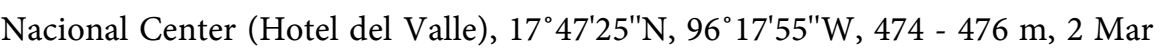
2008, T. Croat 100128 (MO, XAL); Municipio Santa María Chimalapa, near of Arroyo Sangre, ca. 2 km E de Santa María, 1654'30"N, 9440'30"W, 280 m, 27 Aug 1984, H. Hernández 369 (CHAPA, MEXU); Chiltepec, Tuxtepec, 7 Aug 1965, G. Martínez 174 (MEXU); Sierra de Juárez, Municipio Valle Nacional, Yetla, km 154 of trail Tuxtepec-Oaxaca, 800 m, 15 Jun 1966, G. Martínez 879 (MEXU, XAL); Distrito Tuxtepec, Municipio Valle Nacional, along hwy México 175, $21 \mathrm{~km}$ (by road) $\mathrm{N}$ of Vista Hermosa and $4 \mathrm{~km} \mathrm{SW}$ of Valle Nacional, $17^{\circ} 44^{\prime} \mathrm{N}, 96^{\circ} 21^{\prime} \mathrm{W}, 450 \mathrm{~m}, 15 \mathrm{Dec}$ 1985, M. Nee 32158 (NY, UC, XAL); Municipio Putla, Distrito Putla, ca. 4 km NE of San Pedro, 800 m, 11 Jul 1988, E. Solano 429 (CHAPA); Municipio Santa María Jacatepec, Distrito Tuxtepec, trail to Cosolapa San Antonio, Ejido San Felipe Tilpa, $13.3 \mathrm{~km}$ SW of La Reforma, $17^{\circ} 51^{\prime} \mathrm{N}, 96^{\circ} 03^{\prime} \mathrm{W}, 300$ m, 20 Feb 1988, R. Torres \& L. Cortes 11439 (MEXU); Municipio Matías Romero, hills between Río Verde and Arroyo Hamaca, near Río Verde, $10 \mathrm{~km}$ SE from the La Foresta sawmill (abandoned), ca. $24 \mathrm{~km} \mathrm{~S}$ of Esmeralda, $17^{\circ} 01^{\prime} \mathrm{N}, 94^{\circ} 44^{\prime} \mathrm{W}, 300$ m, 27 Sep 1982, T. Went et al. 4007 (CHAPA, UC); Santa María Chimalapa, Arroyo Sangre, ca. 1 - $2 \mathrm{~km}$ W of Santa María, $16^{\circ} 54^{\prime} 30^{\prime \prime} \mathrm{N}, 94^{\circ} 40^{\prime} \mathrm{W}, 250 \mathrm{~m}, 27 \mathrm{Jul}$ 1985, T. Went et al. 5132 (MEXU). Puebla: Municipio Hueytamalco, $1 \mathrm{~km}$ NE of Campo Experimental "Las Margaritas" facilities, Instituto Nacional de Investigaciones Forestales, Agrícolas y Pecuarias (INIFAP), $20^{\circ} 00^{\prime} 25^{\prime \prime} \mathrm{N}, 97^{\circ} 18^{\prime} 45^{\prime \prime} \mathrm{W}, 490 \mathrm{~m}, 16$ Jun 2007, G. Ibarra et al. 5333 (MEXU). Tabasco: Municipio Tacotalpa, Cerro del Madrigal, km 7 of Tacotalpa station to Tapijulapa, 13 Apr 1980, C. Cowan 2908 (MEXU); $13 \mathrm{mi} \mathrm{N}$ of Ocozocuautla on gravel road to Apitac, near km 21 marker, 900 m, 9 Jul 1977, T. Croat 40619 (MEXU, MO); Municipio Teapa, surroundings of Centro Regional Puyacatengo, 16 Jan 1982, S. Zamudio 180 (XAL). Veracruz: Municipio Atoyac, Ejido Esperanza, $18^{\circ} 52^{\prime} 00^{\prime \prime N}, 96^{\circ} 44^{\prime} 00^{\prime \prime W}, 750$ m, 21 Jun 1985, R. Acevedo \& G. Castillo 281 (XAL); Municipio Atoyac, Miraflores, 1857'00"N, 96 49'00"W, 750 m, 22 Jun 1985, R. Acevedo \& G. Castillo 286 (MEXU); Municipio San Andrés Tuxtla, Estación de Biología Tropical Los Tuxtlas, 150 m, 5 Oct 1974, J. Calzada 1547 (XAL); Municipio Catemaco, hills S of Cerro Jegal, N of Los Naranjos Ranch, road entrance Catemaco-Dos Amates, 21 May 1985, J. Calzada 11575 (XAL); Municipio Soteapan, Ejido Piedra Labrada to N, 17 May 1985, J. Calzada et al. 11518 (XAL); Municipio Catemaco, El Naranjillo, El Naranjillo Ranch, road Catemaco-Sontecomapan, 18²5'N, 9506'W, 7 Aug 1993, G. Carmona 60 
(XAL); Municipio San Andrés Tuxtla, El Bastonal, 18 Sep 1985, R. Cedillo 3402 (MEXU, XAL); Municipio Jesús Carranza, km 6 from trail Cedillo-Río Alegre, $17^{\circ} 10^{\prime} \mathrm{N}, 94^{\circ} 40^{\prime} \mathrm{W}, 150 \mathrm{~m}, 18$ Jan 1975, J. Dorantes 3916 (XAL); Municipio Catemaco, Coyame, near Catemaco, $18^{\circ} 27^{\prime} \mathrm{N}, 95^{\circ} 05^{\prime} \mathrm{W}, 180 \mathrm{~m}, 22$ Jan 1972, $R$. Hernández 1386 (MEXU, XAL); Municipio Catemaco, Ejido Balzapote, ca. $3 \mathrm{~km}$ $\mathrm{N}$ of the Los Tuxtlas Biological Station, 15 Oct 1992, P. Hietz \& U. Seifert 562 (MEXU); Municipio San Andrés Tuxtla, Laguna Escondida, $5 \mathrm{~km} \mathrm{NE}$ of Estación de Biología Tropical Los Tuxtlas, 18³4 - 36'N, $95^{\circ} 04$ - 09'W, 250 m, 12 Sep 1982, G. Ibarra 307 (MEXU); Municipio Montepio, Estación de Biología Tropical Los Tuxtlas, $18^{\circ} 34^{\prime} \mathrm{N}, 94^{\circ} 05^{\prime} \mathrm{W}$, ca. 140 m, 29 Jul 1976, H. Kennedy \& $C$. Horvitz 3676 (MEXU); 33 km NE of Catemaco, Biological Station, $200 \mathrm{~m}, 30$ Apr 1973, K. Kramer 1974 (MEXU); Municipio San Andrés Tuxtla, Estación de Biología Tropical Los Tuxtlas, Cerro Vigia 5, lot 67, $18^{\circ} 34^{\prime} \mathrm{N}, 95^{\circ} 04^{\prime} \mathrm{W}, 450 \mathrm{~m}, 12$ Apr 2005, T. Krömer et al. 1924 (MEXU); Municipio San Andrés Tuxtla, Colegio Agrícola Militar, Montepio “Rancho Pouchoulen”, 18³4'N, 9508'W, 740 m, 28 Apr 2005, T. Krömer et al. 2029 (MEXU, XAL); Municipio Catemaco, La Palma, Don Fidel plot, $18^{\circ} 33^{\prime} \mathrm{N}, 95^{\circ} 04^{\prime} \mathrm{W}, 35$ m, 8 Aug 2005, T. Krömer et al. 2465 (MEXU); Municipio Catemaco, $5 \mathrm{~km}$ E of Tebanca course to Bastonal, N slope of Sierra de Santa Marta, 830 m, 10 Dec 1980, R. Lira 170 (XAL); Municipio Las Choapas, "El Milagro" Ranch, $5 \mathrm{~km}$ in a straight line to SW $\left(202^{\circ}\right)$ of Nueva Tabasqueña colony, 17³1'48"N, 9401'44"W, 115 m, 27 Aug 2002, E. López 241 b (XAL); Municipio San Andrés Tuxtla, Estación de Biología Tropical Los Tuxtlas, between Montepio and Sontecomapan, 150 m, 12 Jun 1981, D. Lorence 3477 (XAL); Municipio Catemaco, ca. $8 \mathrm{~km}$ after Coyame course to Adolfo López Mateos, $18^{\circ} 26^{\prime} 22^{\prime \prime N}, 95^{\circ} 00^{\prime} 11^{\prime \prime} \mathrm{W}, 650 \mathrm{~m}, 3$ May 2002, A. Mendoza et al. $617 \mathrm{~b}$ (MEXU); Municipio Pajapan, $5 \mathrm{~km}$ NW of Pajapan, SE slopes of Cerro San Martín Pajapan, $18^{\circ} 17^{\prime} 30^{\prime} \mathrm{N}, 94^{\circ} 43^{\prime} \mathrm{W}, 700$ m, 3 Nov 1981, M. Nee \& J. Calzada 22768 (MEXU, XAL); gravel Cliff $1 \mathrm{mi}$ W of Fortín, 4 Aug 1947, J. Paxson et al. $17 M 680$ (MEXU, UC); Municipio San Andrés Tuxtla, Colegio Agrícola Militar, Montepio, Huber Ranch, 18 $36^{\prime} \mathrm{N}, 95^{\circ} 05^{\prime} \mathrm{W}, 70$ m, 7 Sep 2005, A. Pérez et al. 11 (MEXU); Municipio San Andrés Tuxtla, Estación de Biología Tropical Los Tuxtlas, 28 Oct 1981, T. Ramamoorthy 2613 (MEXU); Municipio San Andrés Tuxtla, Estación de Biología Tropical Los Tuxtlas, 19 Jan 1982, T. Ramamoorthy \& G. Ibarra 3268 (MEXU); Municipio Catemaco, Península de Moreno, tral Coyame-Península de Moreno, 18 $27^{\prime} \mathrm{N}, 95^{\circ} 00^{\prime} \mathrm{W}, 200$ m, 18 Aug 1980, F. Ramírez 1382 (XAL); Municipio Catemaco, trail Tebanca-El Bastonal, $18^{\circ} 24^{\prime} \mathrm{N}$, 9459'W, 400 m, 27 mar 1980, F. Ramírez \& F. Vázquez 704 (XAL); Municipio Catemaco, Santa Rosa, $18 \mathrm{~km} \mathrm{~S}$ of Catemaco, 450 m, 10 Apr 1973, R. Riba et al. 705 (MEXU); San Pedro Soteapan, Arroyo Soteapan, $1 \mathrm{~km}$ W ofSan Fernando, $S$ slope of Sierra de Santa Marta, 600 m, 13 Aug 1980, R. Riba et al. 1135 (MEXU); Municipio Catemaco, Península de Moreno, ca. 4 km E of Coyame, 300 m, 17 ago 1980, R. Riba et al. 1223 (MEXU); Municipio Coetzala, hillside E of Coetzala, $18^{\circ} 46^{\prime} 50^{\prime \prime} \mathrm{N}, 96^{\circ} 54^{\prime} 52^{\prime \prime} \mathrm{W}, 650 \mathrm{~m}, 4$ Nov 2001, A. Rincón \& C. Durán 2819 (XAL); Municipio Catemaco, Cerro Jegal, $8 \mathrm{~km} \mathrm{~N}$ of Catemaco, 18 $29^{\prime} 05^{\prime \prime} \mathrm{N}$, 
9503'53"W, 940 m, 4 nov 2000, A. Rincón et al. 2137 (MEXU, XAL); some km before Montepio, $18^{\circ} 38^{\prime} \mathrm{N}, 95^{\circ} 05^{\prime} \mathrm{W}, 2$ May 1988, J. van Rooden 810 (MEXU); Municipio Catemaco, Dos Amates de Sontecomapan, 4 Aug 1965, 500 m, J. Rzedowski 20356 (MEXU); Municipio Amatlán, Cerro Cruztitlán, near the cave chapel of La Virgen, in front of Peñuelas, E of Amatlán, $18.84704^{\circ} \mathrm{N}$, 96.88715 W, 743 m, 30 Jan 2015, G. Salazar et al. 9376 (MEXU); Municipio Catemaco, at highest point on road from Catemaco to Sontecomapan, $5 \mathrm{~km} \mathrm{~N}$ of juntion with road around Lago Catemaco, $8 \mathrm{~km}$ (by air) NE of Catemaco, $18^{\circ} 29^{\prime} \mathrm{N}, 95^{\circ} 04^{\prime} \mathrm{W}, 510 \mathrm{~m}, 14$ Jan 1981, G. Schatz \& M. Nee 227 (XAL); Municipio Catemaco, Dos Amates, Cerro Chochobi, 720 m, 20 Dec 2006, B. Senterre \& M. Parvais 4379 (XAL); Municipio San Andrés Tuxtla, Estación de Biología Tropical Los Tuxtlas, $18^{\circ} 34^{\prime} \mathrm{N}-18^{\circ} 36^{\prime} \mathrm{N}, 95^{\circ} 04 \mathrm{~W}-95^{\circ} 09^{\prime} \mathrm{W}, 700 \mathrm{~m}, 10$ Sep 1986, S. Sinaca 921 (MEXU); Municipio San Andrés Tuxtla, Ejido Balzapote, $5 \mathrm{~km} \mathrm{~N}$ of Estación de Biología Tropical Los Tuxtlas, $18^{\circ} 34^{\prime} \mathrm{N}-18^{\circ} 36^{\prime} \mathrm{N}$, $95^{\circ} 04^{\prime} \mathrm{W}$ - 95 09'W, $180 \mathrm{~m}, 16$ Feb 1989, S. Sinaca \& P. Ventura 1490 (MEXU); 3 $\mathrm{km} \mathrm{N}$ of Catemaco, along dirt road, $18^{\circ} 30^{\prime} \mathrm{N}, 95^{\circ} 10^{\prime} \mathrm{W}, 400 \mathrm{~m}, 15$ Jun 1982, $S$. Solheim \& V. Powers 848 (XAL); Municipio Catemaco, Estación de Biología Universidad de Veracruz, 500 - 650 m, 14 Sep 1998, A. Torrez \& A. Campos 18 (MEXU); Municipio Hidalgotitlán, E of Campamento Hermanos Cedillo to La Laguna, $1 \mathrm{~km}$ of camp, 11 Apr 1974, P. Valdivia 212 (XAL); Municipio Hidalgotitlán, 2, $5 \mathrm{~km}$ of Campamento Hermanos Cedillo in front of Paso Moral, N side, $17^{\circ} 15^{\prime} 30^{\prime \prime} \mathrm{N}, 94^{\circ} 36^{\prime} \mathrm{W}, 150 \mathrm{~m}, 18$ Apr 1974, P. Valdivia 324 (XAL); Municipio Hidalgotitlán, to Álvaro Obregón, $6 \mathrm{~km}$ of Campamento Hermanos Cedillo, $17^{\circ} 18.7^{\prime} \mathrm{N}, 94^{\circ} 39^{\prime} \mathrm{W}, 149$ m, 23 Apr 1974, P. Valdivia 416 (XAL); Municipio Hidalgotitlán, hacia Álvaro Obregón, $6 \mathrm{~km}$ of Campamento Hermanos Cedillo, $17^{\circ} 18,7^{\prime} \mathrm{N}, 94^{\circ} 39^{\prime} \mathrm{W}, 150 \mathrm{~m}, 23$ Apr 1974, P. Valdivia 426 (XAL); Municipio Hidalgotitlán, to Álvaro Obregón, $300 \mathrm{~m}$ of Campamento Hermanos Cedillo, by Plan de Arroyo, W direction, 17 $16^{\prime} \mathrm{N}, 94^{\circ} 37^{\prime} \mathrm{W}, 149$ m, 24 Apr 1974, P. Valdivia 452 (XAL); Municipio Hidalgotitlán, $6.5 \mathrm{~km}$ of Campamento Hermanos Cedillo to La Laguna, $17^{\circ} 16.6^{\prime} \mathrm{N}, 94^{\circ} 33.4^{\prime} \mathrm{W}, 159 \mathrm{~m}, 23$ May 1974, $P$. Valdivia 615 (XAL); Municipio Hidalgotitlán, in $\mathrm{km} 6.06$ and to $200 \mathrm{~m}$ of $\mathrm{N}$ side course to La Laguna, $17^{\circ} 16.3^{\prime} \mathrm{N}, 94^{\circ} 35.2^{\prime} \mathrm{W}, 160 \mathrm{~m}, 28$ May 1974, P. Valdivia 760 (XAL); Municipio Hidalgotitlán, $1.7 \mathrm{~km}$ of Campamento Hermanos Cedillo to La Escuadra, $17^{\circ} 17^{\prime} \mathrm{N}, 94^{\circ} 36.5^{\prime} \mathrm{W}, 156$ m, 22 Jun 1974, P. Valdivia 915 (XAL); Municipio Hidalgotitlán, $1.5 \mathrm{~km}$ of Campamento Hermanos Cedillo to La Escuadra, $17^{\circ} 16.5^{\prime} \mathrm{N}, 94^{\circ} 35.3^{\prime} \mathrm{W}, 156 \mathrm{~m}, 24$ Jun 1974, P. Valdivia 943 (XAL); Municipio Hidalgotitlán, $800 \mathrm{~m}$ of Campamento Hermanos Cedillo to La Escuadra, $17^{\circ} 15^{\prime} \mathrm{N}, 94^{\circ} 37^{\prime} \mathrm{W}, 156$ m, 22 Jul 1974, P. Valdivia 995 (XAL), ibídem, 24 Jul 1974, P. Valdivia 1075 (XAL); Municipio Hidalgotitlán, $1 \mathrm{~km}$ of Campamento Hermanos Cedillo to La Escuadra, 17 $15^{\prime} \mathrm{N}, 94^{\circ} 37^{\prime} \mathrm{W}, 156 \mathrm{~m}, 29$ Jul 1974, P. Valdivia 1164 (XAL); Municipio Hidalgotitlán, $2 \mathrm{~km}$ of Campamento Hermanos Cedillo to La Escuadra, $17^{\circ} 17.3^{\prime} \mathrm{N}, 94^{\circ} 36.5^{\prime} \mathrm{W}, 156 \mathrm{~m}$, 31 Jul 1974, P. Valdivia 1202 (XAL), ibídem, 01 Aug 1974, P. Valdivia 1229 (XAL), ibídem, $P$. Valdivia 1242 (XAL); Municipio Hidalgotitlán, $1.5 \mathrm{~km}$ of Campamento 
Hermanos Cedillo to La Escuadra, $17^{\circ} 15.5^{\prime} \mathrm{N}, 94^{\circ} 37^{\prime} \mathrm{W}, 150 \mathrm{~m}, 2$ Aug 1974, $P$. Valdivia 1259 (XAL); Municipio Hidalgotitlán, $6 \mathrm{~km}$ of Campamento Hermanos Cedillo to La Escuadra, 17²18,7'N, 94³9'W, 150 m, 06 Aug 1974, P. Valdivia 1292 (XAL); Municipio Hidalgotitlán, La Escuadra, 17¹9'N, 94³8.5'W, 160 m, 07 Aug 1974, P. Valdivia 1325 (XAL); Municipio Hidalgotitlán, $500 \mathrm{~m}$ of Campamento Hermanos Cedillo to La Escuadra, $17^{\circ} 14^{\prime} \mathrm{N}, 94^{\circ} 37^{\prime} \mathrm{W}, 156 \mathrm{~m}, 09 \mathrm{Oct}$ 1974, P. Valdivia 1380 (XAL); Municipio Hidalgotitlán, $6 \mathrm{~km}$ of Campamento Hermanos Cedillo to La Laguna, $17^{\circ} 16^{\prime} \mathrm{N}, 94^{\circ} 32.5^{\prime} \mathrm{W}, 156 \mathrm{~m}, 12$ Aug 1974, $P$. Valdivia 1440 (XAL); Municipio Hidalgotitlán, 500 m of Campamento Hermanos Cedillo to La Escuadra, 17²16.5'N, 94 37.5'W, 156 m, 14 ago 1974, P. Valdivia 1469 (XAL); Municipio Hidalgotitlán, a $1.5 \mathrm{~km}$ of Campamento Hermanos Cedillo to La Escuadra, $17^{\circ} 15.5^{\prime} \mathrm{N}, 94^{\circ} 37^{\prime} \mathrm{W}, 156$ m, 13 Dec 1974, P. Valdivia 1600 (XAL); Municipio Hidalgotitlán, $4.5 \mathrm{~km}$ of Campamento Hermanos Cedillo to La Escuadra, $17^{\circ} 16.5^{\prime} \mathrm{N}, 94^{\circ} 38^{\prime} \mathrm{W}, 149 \mathrm{~m}, 19 \mathrm{Dec}$ 1974, P. Valdivia 1706 (XAL); Municipio Hidalgotitlán, $3 \mathrm{~km}$ of Campamento Hermanos Cedillo to La Escuadra, $17^{\circ} 16.5^{\prime} \mathrm{N}, 94^{\circ} 37.8^{\prime} \mathrm{W}, 155 \mathrm{~m}, 21$ Jan 1975, P. Valdivia 1776 (XAL); Municipio Hidalgotitlán, $7 \mathrm{~km}$ going Río Alegre, $17^{\circ} 12^{\prime} \mathrm{N}, 95^{\circ} 04^{\prime} \mathrm{W}, 120 \mathrm{~m}, 26$ Jan 1975, $P$. Valdivia 1888 (XAL); Municipio Hidalgotitlán, $4 \mathrm{~km}$ of Campamento Hermanos Cedillo to La Laguna, $17^{\circ} 16^{\prime} \mathrm{N}, 94^{\circ} 35^{\prime} \mathrm{W}, 160 \mathrm{~m}, 28$ Jan 1975, P. Valdivia 1904 (XAL); Municipio Jesús Carranza, $10 \mathrm{~km} \mathrm{~S}$ of Hermanos Cedillo, road to Río Alegre, $17^{\circ} 12^{\prime} \mathrm{N}, 94^{\circ} 41^{\prime} \mathrm{W}, 150$ m, 30 May 1974, M. Vázquez 708 (XAL); Estación de Biología Tropical Los Tuxtlas, $19 \mathrm{mi}$ (by road) NW of Catemaco, $18^{\circ} 35^{\prime} \mathrm{N}$, $95^{\circ} 04^{\prime} \mathrm{W}, 400 \mathrm{ft}$ [=122 m], 17 Aug 1976, G. Webster \& W. Armbruster 20888 A (MEXU); Municipio Jesús Carranza, hills $\mathrm{S}$ of Pob. 2, (near $3 \mathrm{~km} \mathrm{~S}$ Of the junction of the earth trail La Laguna-Sarabia with the trail $\mathrm{N}$ to Pob. $2,17^{\circ} 12^{\prime} \mathrm{N}$, $94^{\circ} 39^{\prime} \mathrm{W}, 250 \mathrm{~m}, 14 \mathrm{Jul}$ 1984, T Went \& A. Villalobos 4454 (CHAPA, MEXU); Municipio Hidalgotitlán, $\mathrm{N}$ of La Laguna camp, near and $\mathrm{N}$ Of the rubber plantation and the $\mathrm{W}$ of Río Cuevas, $17^{\circ} 17^{\prime} 15^{\prime \prime} \mathrm{N}, 94^{\circ} 30^{\prime} 45^{\prime \prime} \mathrm{W}, 125 \mathrm{~m}, 25$ Sep 1980, T. Went et al. 2739 (CHAPA, MEXU).

Campyloneurum madrense differs from $C$. angustifolium by not pruinose (vs. pruinose) rhizome or scarcely so, linear (vs. lanceolate) and narrower (0.4 - 0.8 $\mathrm{mm}$ vs. 1.0 - $1.7 \mathrm{~mm}$ ) rhizome scales with sparsely denticulate (vs. entire) margin, they dark gray to blackish (vs. yellowish-brown to grayish-brown), inside with narrow oblong (isodiametric) cells and present at lower (25 - $940 \mathrm{~m}$ vs. (700-) $1200-1600(-2000) \mathrm{m})$ altitudinal distribution. Also the new species differs from $C$. gracile A. Rojas by thicker (2 - $5 \mathrm{~mm}$ vs. $1.5-2.5 \mathrm{~mm}$ ) rhizome, longer (3 - $6 \mathrm{~mm}$ vs. 1.5 - $3.0(-4.0) \mathrm{mm}$ ) rhizome scales with longer (2 - 5 times longer than wide vs. 1-2 times) cells and relative longer ((18-) $30-65 \mathrm{~cm}$ vs. (10-) $18-40(-55) \mathrm{cm}$ ) fronds (see Figure 3 and Table 3 ).

Studies on the type material of Campyloneurum angustifolium (Swartz s.n., holotype: S!; isotype: BM!), particularly on the isotype in BM is evident that this species has pruinose rhizome and yellowish-brown to brown rhizome scales, and also the material identified as $C$. ensifolium in Mexico not is significantly different, only in shorter and obtuse rhizome scales (present in the northernmost 


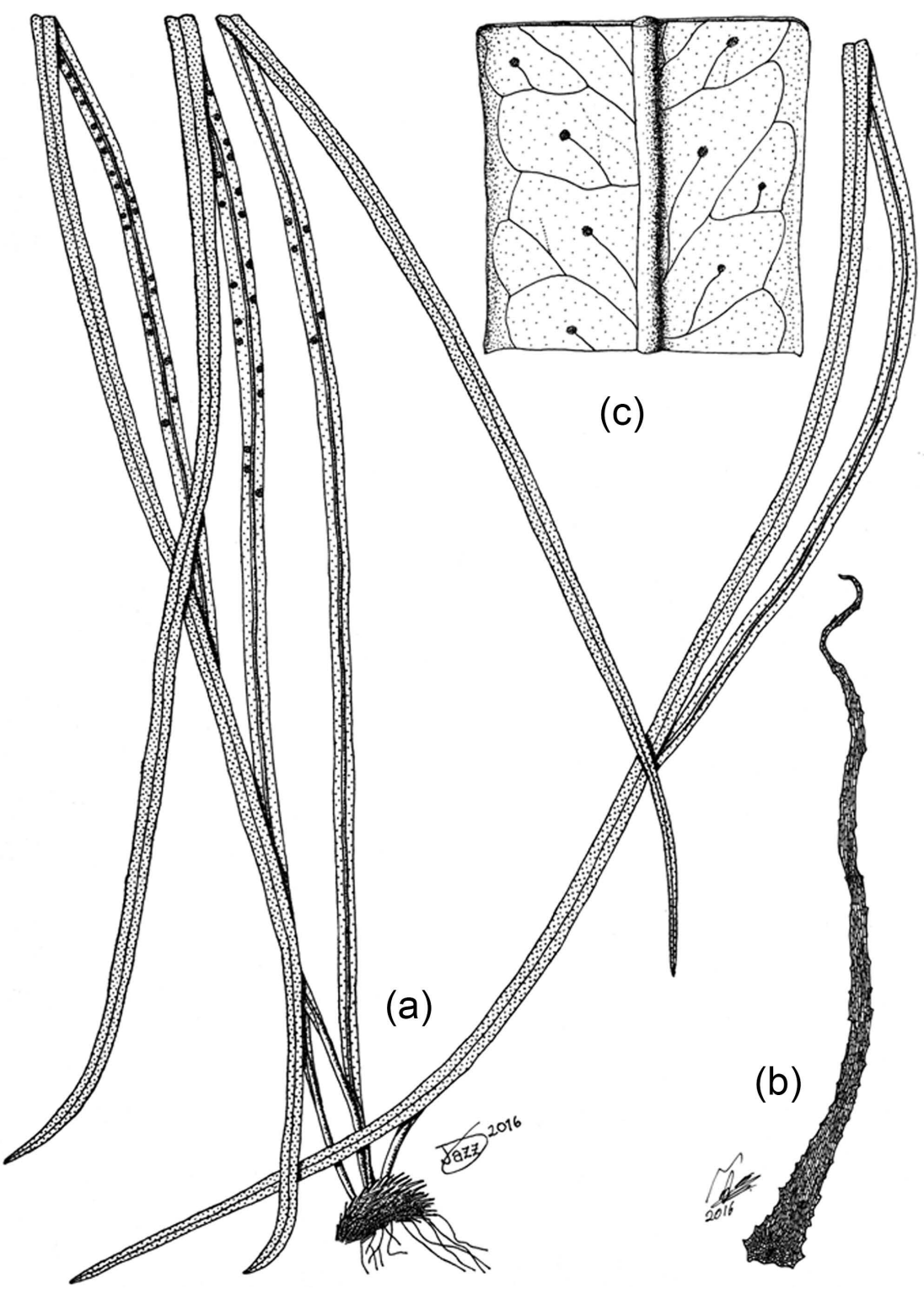

Figure 3. (a) (b). Type material of Campyloneurum madrense A. Rojas (D. Alvarez et al. 6960, MEXU). (a) Habit; (b) Rhizome scales; (c) Blade detail.

populations in Mexico), but this parameter is variable in the species gradually changing to South. However, Mickel \& Smith mentioned in the key and description of $C$. angustifolium from Mexico with oblong cells in rhizome scales and they twice or more longer than wide, characters more related with $C$. madrense, but also they mentioned populations of $C$. angustifolium in South of Mexico that are present at lower elevation and that have narrow rhizome scales with small cell lumen, and they associated this entity with $C$. angustipaleatum [7], a species present in Costa Rica to Bolivia at high elevations.

\subsection{Accepted Name and Lectotype Designed}

\section{Campyloneurum pittieri}

(Christ) Ching, Sunyatsenia 5: 263. 1940 (Figure 4). 
Table 3. Comparison of morphological characteristics among three species of Campyloneurum: C. angustifolium, C. gracile and C. madrense.

\begin{tabular}{|c|c|c|c|}
\hline Character & C. angustifolium & C. gracile & C. madrense \\
\hline Diameter of rhizome & $2-5 \mathrm{~mm}$ & $1.5-2.5 \mathrm{~mm}$ & $2-5 \mathrm{~mm}$ \\
\hline Pruinose rhizome & yes & not & not or scarcely so \\
\hline Distance between fronds & $\begin{array}{c}\text { approximate to } 5 \mathrm{~mm} \\
\text { distant }\end{array}$ & approximate & $\begin{array}{c}\text { approximate to } 2 \\
\text { mm distant }\end{array}$ \\
\hline Long of rhizome scales & $3-5 \mathrm{~mm}$ & $1.5-3.0(-4.0) \mathrm{mm}$ & $3-6 \mathrm{~mm}$ \\
\hline Broad of rhizome scales & $1.0-1.7 \mathrm{~mm}$ & $0.5-1.0 \mathrm{~mm}$ & $0.4-0.8 \mathrm{~mm}$ \\
\hline Margin of rhizome scales & entire & $\begin{array}{c}\text { entire to } \\
\text { denticulate }\end{array}$ & denticulate \\
\hline Color of rhizome scales & $\begin{array}{l}\text { yellowish-brown to } \\
\text { grayish-brown }\end{array}$ & brown to dark brown & $\begin{array}{c}\text { dark gray to } \\
\text { blackish }\end{array}$ \\
\hline $\begin{array}{l}\text { Form of apical cells of } \\
\text { rhizome scales }\end{array}$ & poligonal, elongate & poligonal, isodiametric & $\begin{array}{l}\text { rectangular to } \\
\text { oblong }\end{array}$ \\
\hline Long of cell of rhizome scales & $\begin{array}{c}1.5 \text { - } 3.0 \text { times longer } \\
\text { than wide }\end{array}$ & $\begin{array}{c}1 \text { - } 2 \text { times longer than } \\
\text { wide }\end{array}$ & $\begin{array}{c}2 \text { - } 5 \text { times longer } \\
\text { than wide }\end{array}$ \\
\hline $\begin{array}{l}\text { Lumen color of } \\
\text { rhizome scales }\end{array}$ & translucid & brown & gray \\
\hline Frond size & $20-45(-75) \mathrm{cm}$ & $(10-) 18-40(-55) \mathrm{cm}$ & $(18-) 30-65 \mathrm{~cm}$ \\
\hline Diameter of stipe & $1-2 \mathrm{~mm}$ & $0.7-1.0 \mathrm{~mm}$ & $1-1.5(-2) \mathrm{mm}$ \\
\hline Broad of blade & $0.4-1.5(-2.0) \mathrm{cm}$ & $0.3-1.5(-2.0) \mathrm{cm}$ & $(0.5-) 0.8-1.5 \mathrm{~cm}$ \\
\hline Altitudinal distribution & $\begin{array}{c}(700-) 1200-1600 \\
(-2000) \mathrm{m}\end{array}$ & $0-900(-1200) \mathrm{m}$ & $25-940 m$ \\
\hline
\end{tabular}

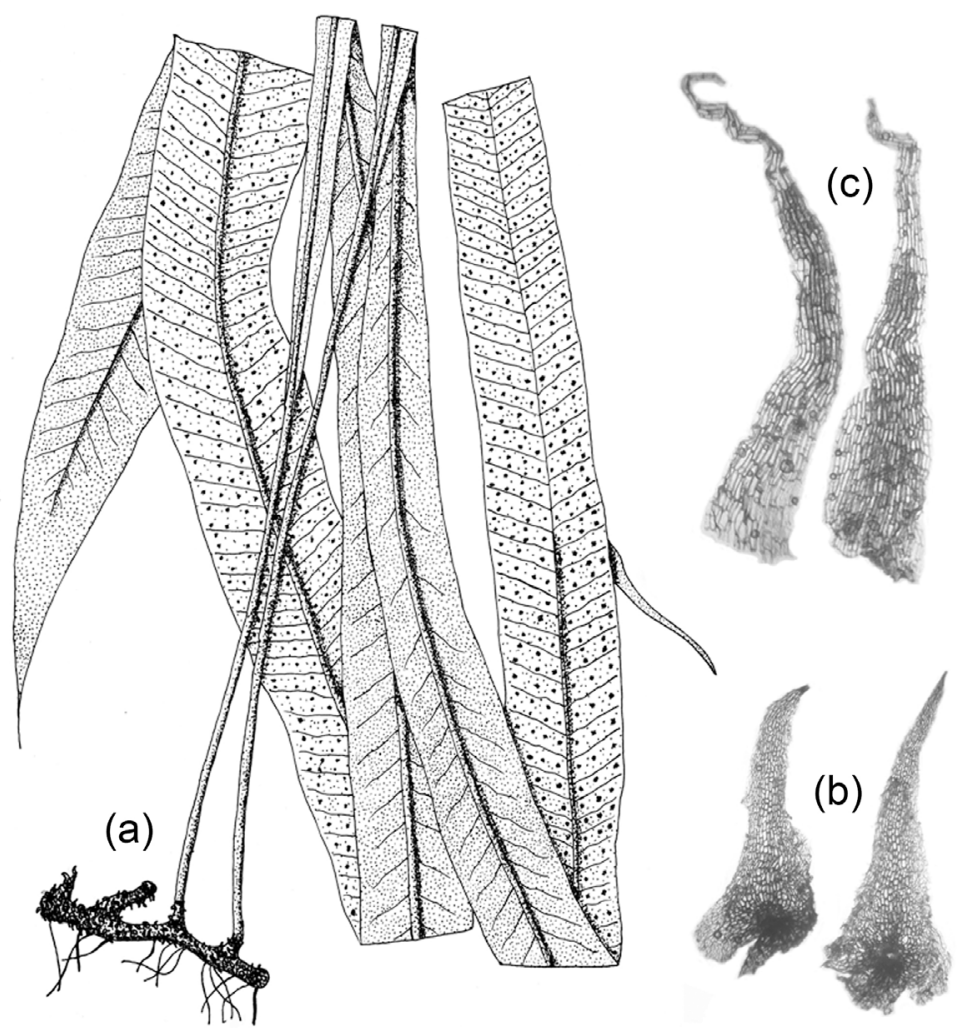

Figure 4. (a) (b). Representative specimen of Campyloneurum pittieri (Christ) Ching ( $A$. Rodríguez \& L. Vargas 3152, CR). (a) Habit; (b) Rhizome scales; (c) Rhizome scales of Campyloneurum amphostenon (Kunze ex Klotzsch) Fée (J. Bittner 109, CR). 
Polypodium pittieri Christ, Prim. Fl. Costaric. 3(1): 16. 1901. Tipo: COSTA RICA. San José: El Páramo, E side of Cerro Buena Vista, 3000 m, Jan 1897, $H$. Pittier 10479 (lectotype designed here: CR!; isolectotypes: BM (photo, GH), BR, P!, US).

Description: Terrestrial; rhizome $2-5 \mathrm{~mm}$ in diameter, creeping, fronds 5 $30(-50) \mathrm{mm}$ distant between them, pruinose; rhizome scales $2-5 \times 0.7-1.5$ $\mathrm{mm}$, ovate-lanceolate, clathrate, grayish-brown, sparse, peltate or with overlapping base, cells rounded to polygonal, lumen translucid; fronds $40-80(-100) \mathrm{cm}$ long, moderately decumbent; stipe $1 / 3-1 / 6$ of the frond length, strawish to pale brown, glabrous, phyllopodia $2-5 \mathrm{~mm}$ long; blade $30-60(-80) \times 2.5-3(-5)$ $\mathrm{cm}$, narrowly elliptic, long acuminate at apex, gradually reduced at base, coriaceous, lustrous; rachis glabrous, pale brown, pale as lamina, principally with immersed lateral veins or inconspicuous; sori rounded, in 3 - 7 rows between costa and margin.

Distribution: Known from Costa Rica and Panama at $700-1800(-2000) \mathrm{m}$ elevation.

Additional revised specimens: COSTA RICA. Cartago: Cerro Asunción, ca. $0.5 \mathrm{~km} \mathrm{NE}$ of the Interamerican Hwy (km 90 - 91), 6 Mar 1986, $3080 \mathrm{~m}, M$. Kappelle 1414 (CR); Volcán Turrialba, outside the crater, 3200 - 3300 m, 16 Jun 1993, K. Mehltreter 432 (CR); El Guarco, Reserva Forestal Los Santos, Savegre basin, over Interamerican road, rute to Cerro de La Muerte, between $\mathrm{km} 78$ and 79. $9^{\circ} 37^{\prime} 05^{\prime \prime N}, 83^{\circ} 49^{\prime} 0 " \mathrm{~W}, 2960 \mathrm{~m}, 28$ Jan 1998, A. Rodríguez et al. 2971 (INB, CR, MO); Volcán Turrialba, high part of the hill, 31 ene 1957, R. Rodríguez 98 (CR); Paraíso, Valle del Reventazón, Cerros Sákira, Sábila and Zacatales, $9^{\circ} 35^{\prime} 20^{\prime \prime N}$, 834ㄴ'40"W, 3300 - 3420 m, 16 Jul 1996, A. Rojas \& M. Coto 2764 (INB, MO); Cerros Cuericí, 2940 m, 9 Nov 1988, Y. Widmer 395 (CR). Cartago-San José: ca. $22 \mathrm{~km}$ SE of El Empalme, along Interamerican Hwy, 2500 - 2600 $\mathrm{m}, 9^{\circ} 40^{\prime} \mathrm{N}, 83^{\circ} 50^{\prime} \mathrm{W}, 27$ Nov 1969, W. Burger \& R. Liesner 6501 (CR, F); just below of highest point on the Interamerican hwy, NW of La Asunción, $9^{\circ} 34^{\prime} \mathrm{N}$, $83^{\circ} 45^{\prime} \mathrm{W}, 3000$ - 3200 m, 5 Feb 1982, W. Burger \& K. Barringer 11506 (CR, F); Cordillera de Talamanca, upper slopes, $\mathrm{W}$ ridge of Cerros Cuericí, ca. $9^{\circ} 34^{\prime} \mathrm{N}$, $83^{\circ} 40^{\prime} \mathrm{W}, 3160$ m, 15 Sep 1983, G. Davidse 24661 (CR, MO); Cordillera de Talamanca, Cerros Cuericí, Parque Nacional Chirripó, continental divide, $9^{\circ} 35^{\prime} \mathrm{N}$, 83³8'30"W, 3200 m, 16 Sep 1983, G. Davidse 24813 (CR, MO); Villa Mills, 2 Aug 1961, D. Knight s.n. (CR). Heredia: Parque Nacional Braulio Carrillo, volcán Barva station, path and forest between park office and crater, $10^{\circ} 07^{\prime} 20^{\prime \prime} \mathrm{N}$, 84 06'00"W, 2700 - 2900 m, 13 Jul 1991, H. Gay et al. 1511 (CR); hills SW of Volcán Barba, 2700 - 2900 m, Apr 1971, L. Gómez \& W. Wagner s.n. (CR); slopes and ridges above Laguna del Barva and Summit of Volcán Barva, $10^{\circ} 08^{\prime} \mathrm{N}$, $84^{\circ} 06,5^{\prime} \mathrm{W}, 2840$ - 2900 m, 27 Apr 1986, M. Grayum \& F. Quesada 7424 (CR, MO); Volcán Barva, trail to Laguna Copey, 2700 - 2800 m, 24 Jun 1993, K. Mehltreter 467 (CR); Barva, Parque Nacional Braulio Carrillo, Tárcoles basin,

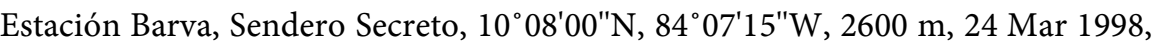
A. Rodríguez \& L. Vargas 3152 (CR, INB, MO); Barva, Parque Nacional Braulio Carrillo, Sarapiquí basin, surroundings of the path that goes to the viewpoint, 
$10^{\circ} 08^{\prime} 10^{\prime \prime} \mathrm{W}, 84^{\circ} 06^{\prime} 25^{\prime \prime} \mathrm{W}, 1900$ m, 2 Jun 1998, A. Rodríguez \& L. Vargas 3456 (CR, INB, MO); road from Cartago to San Isidro del General, (Pan American Hwy, Rte 2), km 87 - 88, ca. 1 km NW of Asunción, 3200 m, 29 Jan 1986, A. Smith \& T. Béliz 2022 (CR, UC); Aromal del Volcán Barba, 2700 m, 8 May 1927, M. Valerio 64 (CR). Limón: Cordillera de Talamanca, Atlantic slope, Kámuk massif, paramo $\mathrm{NE}$ of the main Kámuk peak, $9^{\circ} 16^{\prime} \mathrm{N}-9^{\circ} 17^{\prime} \mathrm{N}, 83^{\circ} 00^{\prime} \mathrm{W}$ $83^{\circ} 02^{\prime} \mathrm{W}, 3000$ - 3300 m, 17 - 18 Sep 1984, G. Davidse \& G. Herrera 29328 (CR, MO); cordillera de Talamanca, peak of Cerro Kámuk, $9^{\circ} 16^{\prime} 30^{\prime \prime} \mathrm{N}, 83^{\circ} 02^{\prime} \mathrm{W}, 3350$ - 3549 m, 25 Mar 1984, G. Davidse et al. 26019 (CR, MO); Chirripó National Park, N end of Loma Larga, ca. 3350 m, 15 Feb 1983, N. Garwood et al. 1263 (CR). Limón-Puntarenas: Cordillera de Talamanca, between Cerro Kasir and Cerro Nai, continental divide, $9^{\circ} 12^{\prime} \mathrm{N}-9^{\circ} 13^{\prime} \mathrm{N}, 83^{\circ} 03^{\prime} 30^{\prime \prime} \mathrm{W}-83^{\circ} 04^{\prime} 30^{\prime \prime} \mathrm{W}, 2900$ 3050 m, 22 Mar 1984, G. Davidse et al. 25837 (CR, MO). San José: Pérez Zeledón, Interamerican hwy ( $\mathrm{km} \mathrm{82)}$ in the near of the Cerro de La Muerte border, $9^{\circ} 34^{\prime} 20^{\prime \prime N}, 83^{\circ} 45^{\prime} 30^{\prime \prime} \mathrm{W}, 3000 \mathrm{~m}, 12$ Jun 1991, J. Bittner 1097 (CR); along the trail from Canaán to Chirripó, via Los Ángeles, above ( $\mathrm{N}$ of) the Río Talari, $9^{\circ} 30^{\prime} \mathrm{N}$, $83^{\circ} 32^{\prime} \mathrm{W}, 3100$ - 3200 m, 24 Aug 1971, W. Burger 8323 (CR, F); along Interamerican Hwy, 940'N, 8350'W, 2840 m, 23 Nov 1986, E. Hennipman et al. 7131 (CR, U); Cerro Las Vueltas, between Cruces and Alto Indio, 3020 m, 11 Mar 1986, M. Kappelle 1622 (CR); Parque Nacional Chirripó, Monte Sin Fé, 3250 m, 20 Sep 1988, M. Kappelle 2674 (CR); Parque Nacional Chirripó, Palmito Morado, 2910 m, 20 Jan 1989, M. Kappelle \& M. Monge 4902 (CR); San Gerardo de Dota, 2800 m, Jun-Jul 1989, H. Van Velzen \& T. Genze 62 (CR).

PANAMÁ. Bocas del Toro: Cordillera de Talamanca, 2 airline $\mathrm{km} \mathrm{NW}$ of the main peak of cerro Fábrega, along the NW ridge of the massif, $9^{\circ} 08^{\prime} \mathrm{N}, 82^{\circ} 53^{\prime} \mathrm{W}$, 3150 - 3200 m, 7 - 8 Mar 1984, G. Davidse et al. 25333 (CR, MEXU, MO); between Itamut \& Bine peaks, Fabrega massiff, 3200 m, 5 - 9 Mar 1984, L. Gómez et al. 22547 (CR); 1 - 2 km SWW of Itamut camp, 3175 m, 6 - 7 Mar 1984, L. Gómez et al. 22615 (CR).

Campyloneurum pittieri differs from C. amphostenon because has not pruinose or scarcely pruinose (vs. pruinose); rhizome scales longer (4 - $8 \mathrm{~mm}$ vs. 2 $5 \mathrm{~mm}$ ), ovate with a long acuminate tip (vs. lanceolate with acute tip), they are with brown to dark brown color (vs. yellowish-brown to grayish-brown) and with the apical cells 1 - 2 times longer than wide (vs. 1.5 - 3 times); fronds relatively longer ((35-) $45-60(-80) \mathrm{cm}$ vs. $30-55(-65) \mathrm{cm})$; stipe longer $(8-25 \mathrm{~cm}$ vs. 4 - $10(-20) \mathrm{cm})$; blade relatively broader $((1.3-) 2-5 \mathrm{~cm}$ vs. $1-3.5 \mathrm{~cm})$; veins prominently marked (vs. not marked or scarcely so) and distributed to relative higher elevation ((1900-) 2700 - 3400 (-3550) m vs. (1800-) 2100 - 2900 (-3200) m) (see Figure 4 and Table 2).

Detailed studies on the type of Campyloneurum amphostenon (Moritz $120 \mathrm{~b}$, $\mathrm{B}$ !, BK!, K!) demonstrated that this material coincides with the second entity proposed by Rojas (2005), while the first corresponds to $C$. pittieri (Christ) Ching ( H. Pittier 10479, CR!), so the recognition and validation of this species are necessary. 


\section{Acknowledgements}

This work was made possible thanks to Universidad Nacional de Costa Rica (UNA) and Sistema Nacional de Áreas de Conservación (SINAC). Thanks also to Herbario Nacional de Costa Rica (CR) and Herbario Nacional de México (MEXU) for providing specimen loans and the space for analyze the specimens. For the good attention and facilitates of the curators in the Mexican herbaria CHAPA, MEXU and XAL. Thanks to Pablo Muñoz Cambronero, Jasmín Mejía Vargas and Mónica Guzmán Morales for the excellent drawings. To the anonymous reviewers for their help; and to all who, in one way or another, made this work possible.

\section{References}

[1] Gómez, L.D. and Arbeláez, A.L. (2009) Flora de Nicaragua, Helechos (Tomo IV). In: Stevens, W.D., Montiel, O.M. and Pool, A., Eds., Monographs in Systematic Botany from the Missouri Botanical Garden, The Missouri Botanical Garden Press, St. Louis, Vol. 116, 34-36.

[2] Moran, R.C. (2011) Géneros Neotropicales de helechos y licófitas. Organización para Estudios Tropicales, San José, 407 p.

[3] Lellinger, D.B. (1988) Some New Species of Campyloneurum and a Provisional Key to the Genus. American Fern Journal, 78, 14-35. https://doi.org/10.2307/1547598

[4] León, B. (1993) A Taxonomic Revision of the Fern Genus Campyloneurum (Polypodiaceae). Doctoral Thesis, Afdelingen for Systematisk-Biologisk Institut, Aarhus Universitet, Aarhus, $104 \mathrm{p}$.

[5] Lellinger, D.B. (1989) The Ferns and Fern-Allies from Costa Rica, Panama, and the Chocó: Psilotaceae through Dicksoniaceae (Pteridologia). American Fern Society, 255-264.

[6] Rojas, A.F. (2005) El complejo de Campyloneurum agustifolium (Sw.) Fée (Polypodiaceae) en Costa Rica. Lankesteriana, 5, 41-48.

[7] Mickel, J.T. and Smith, A.R. (2004) The Pteridophytes of Mexico (Memoirs of the New York Botanical Garden). NYBG Press, Bronx, Vol. 88, 165-172 and 467-468.

[8] León, B. (1995) Campyloneurum. In: Moran, R.C. and Riba, R., Eds., Flora Mesoamericana. Psilotaceae a Salviniaceae, Universidad Nacional Autónoma de México, México, Vol. 1, 333-338.

http://www.mobot.org/mobot/tropicos/meso/homep_sp.htm

[9] Krier, H.P., Rojas, A.F., Smith, A.R. and Schneider, H. (2007) Hyalotrichopteris Is Indeed a Campyloneurum (Polypodiaceae). American Fern Journal, 97, 127-135. https://doi.org/10.1640/0002-8444(2007)97[127:HIIACP]2.0.CO;2

[10] Mickel, J.T. and Beitel, J.M. (1988) Pteridophyte Flora of Oaxaca, México. Memoirs of the New York Botanical Garden, Bronx, Vol. 46, 96-99 and 470.

[11] Smith, A.R. (1981) Flora of Chiapas, Part 2. Pteridophytes. The California Academy of Sciences. San Francisco.

[12] Stolze, R.G. (1981) Ferns and Fern Allies of Guatemala. Part II. Polypodiaceae. Fieldiana Botany, New Series, Vol. 6, 1-522.

[13] Thiers, B. (2016) Index Herbariorum: A Global Directory of Public Herbaria and Associated Staff. New York Botanical Garden's Virtual Herbarium.

http://sweetgum.nybg.org/science/ih/ 
Submit or recommend next manuscript to SCIRP and we will provide best service for you:

Accepting pre-submission inquiries through Email, Facebook, LinkedIn, Twitter, etc. A wide selection of journals (inclusive of 9 subjects, more than 200 journals)

Providing 24-hour high-quality service

User-friendly online submission system

Fair and swift peer-review system

Efficient typesetting and proofreading procedure

Display of the result of downloads and visits, as well as the number of cited articles Maximum dissemination of your research work

Submit your manuscript at: http://papersubmission.scirp.org/

Or contact ajps@scirp.org 